\title{
CircE: An R implementation of Browne's circular stochastic process model
}

\author{
Michele Grassi, Riccardo Luccio, and Lisa Di Blas \\ University of Trieste, Trieste, Italy
}

\begin{abstract}
In confirmatory analysis of whether data have a circumplex structure, Browne's (1992) model has played a major role. However, implementation of this model requires a dedicated program, CIRCUM, because the analysis routine is not integrated in any of the most widely used statistical software packages. Hence, data entry and graphical representation of the results require the use of one or more additional programs. We propose a package for the R statistical environment, termed $\operatorname{CircE}$, that can be used to enter or import data, implement Browne's confirmatory analysis, and graphically represent the results. Using this new software, we put forward a new approach to assess the sustainability of theoretical models when the analysis is carried out at the level of questionnaire items. The CircE package (for either Mac OS X or Windows) and additional files may be downloaded from http://brm.psychonomic-journals.org/content/supplemental.
\end{abstract}

The term circumplex was introduced by Guttman (1954) to describe specific patterns in correlation matrices in which, as one moves diagonally away from the main diagonal, intercorrelations at first decrease, then increase. A visual-form hypothesis concerning the magnitude and direction of associations among the variables postulates that, as association strength decreases, the distance between variables on the circumference of a circle increases. Thereby, a circumplex representation implies that both the strength and direction of associations among variables should depend on the distances between the variables on the circumference of a circle.

Fabrigar, Visser, and Browne (1997) conducted a review of empirical investigations of circumplex data representations in personality and social psychology. They found two dominant methods of analysis: One was based on exploratory principal components/factor analyses, the other on multidimensional scaling. Because circumplex theories are essentially pictorial representations of the relationships among variables, both of these approaches offer the advantage of yielding graphical representations of the variables' circular ordering. However, neither of these approaches directly examines the extent to which the observed data conform to a circumplex structure. In fact, the two methods can only assess the goodness of fit of two- (or multi-) dimensional scaling/common factor solutions.

To overcome this limit, Browne (1992) proposed a covariance structure modeling (CSM) approach for directly testing circumplex structure. (Unless noted otherwise, all discussion of Browne herein relates to Browne, 1992). This approach allows a researcher to examine the extent to which the underlying structure of a sample correlation matrix conforms to a circumplex pattern and to obtain estimates of the locations of the variables on a circle. Browne's CSM approach is based on earlier work by Anderson (1960). Unlike Anderson's approach, however, Browne's can be applied to data in which all variables are positively correlated, as well as to data in which some variables have negative correlations. Thus, Browne's model - unlike Anderson's - may be seen as particularly applicable to personality psychology, where certain personality traits or emotions are expected to be negatively related to each other.

A practical advantage of approaches utilizing principal components/factor analysis and multidimensional scaling is their availability in most major statistical programs. In this connection, Browne's CSM approach can be tested using CIRCUM, a DOS program that includes special subroutines specifically designed for analyzing circumplex data, which are appended to a general algorithm for fitting nonstandard models called AUFIT (Browne \& Du Toit, 1992). The CIRCUM program allows unconstrained as well as equally spaced estimations of variables' spatial positions around $360^{\circ}$. Constraints can also be applied to the amount of unique ${ }^{1}$ variance relative to each variable and to the minimum common score correlation (MCSC) at $180^{\circ}$ of separation. ${ }^{2}$

Nevertheless, this analysis is limited to about 20 variables, and a modified version of CIRCUM to accommodate larger correlation matrices is not of practical use, since it is an older DOS-based program that Windows XP will not allow to run at all. To run this revised CIRCUM program, the user must find a computer with an older operating system or a good emulator or virtual computer. The program loads an input text file containing some setting parameters and the correlation or covariance matrix among the observed variables. This matrix must be inserted manually in a

M. Grassi, grassi.mic@gmail.com 
triangular form, a nontrivial operation; in fact, only the first 80 columns in each line (paragraph) of the text file are read by the program, and each line must end with the diagonal value 1 . Depending on the number of variables used and the decimal positions retained, this operation can be quite complicated. Moreover, graphical solutions for the visualization of the obtained estimates are not directly available, so that again the use of specific external programs is required.

Although Browne's structural model can be regarded as of fundamental importance in the field of personality research, its implementation has several operational limits. Here we present a program in the $\mathrm{R}$ language (R Development Core Team, 2008) that makes Browne's CSM approach available to $\mathrm{R}$ software users and meets the need of integrating the structural analysis performed by CIRCUM with the software used for data insertion. The program is distributed as a package, called CircE, that is available for free (see the supplemental materials, files CircE.1.0.tgz and CircE.1.0.zip) and can be easily installed directly from the $\mathrm{R}$ console. CircE is an easy-to-use tool that offers the same options available in CIRCUM, allowing constraints to be placed on angular distance (i.e., equal spacing among variables), on common score variance (i.e., equal communalities), and on the MCSC value (i.e., MCSC $=-1$ or 0 ). CircE then overcomes at least four of the limits of CIRCUM: First, CircE facilitates the creation of data files, since $\mathrm{R}$ allows the loading of data sets from several commonly used statistical software programs. Results obtained with CircE can then be directly used for further analyses or graphical representations. Second, when the data sets are imported into $\mathrm{R}$, the functions contained in CircE can be directly used; external data files are no longer needed. Third, CircE offers choices of graphical representations to provide information for interpreting results. Finally, the number of variables that can be analyzed is not limited by CircE but by the computer memory.

In the following sections, we begin with a brief review of Browne's circular stochastic process model with a Fourier series (CSPMF). Next, we set out some statistical aspects useful for the implementation of the model. Practical applications are then considered that illustrate how to fit specific circumplex models and the convergence of CircE's solutions with those obtained using CIRCUM; moreover, integrated graphical solutions are presented. We then introduce a new model constraining variables to fall within a specific arc of the circumference. This new model is the major advantage of CircE, since it is particularly useful in testing the structural validity of a psychological measure at the item level, where a model constraining the location of items to be equally spaced around the circle is too restrictive for the practical purposes of psychological testing. To conclude, we describe typical failures of convergence related to non-positive-definite matrices.

\section{Theoretical Background:}

\section{Browne's Circular Stochastic Process Model}

The approach proposed by Browne (1992) for testing circumplex structure involves specifying a nonstandard type of covariance structure model. This explicitly postulates that the underlying structure of intercorrelations can be represented as an ordering along the circumference of an imaginary circle. Like more traditional types of covariance structure models, this circumplex covariance structure model can be fitted to a sample correlation matrix of observed variables. If we consider a single variable $\mathbf{x}$ as a function of its common part, $c$, and a unique part, $u$, we can write

$$
\mathbf{x}=\mu+\zeta(c+u),
$$

where $\mathbf{x}$ is the $1 \times n$ vector of the $n$ values $x, \mu$ is the conformable vector of the expected values of $x, \xi$ is a nuisance parameter, and $c$ and $u$ are the $1 \times n$ vectors of the true score [with unit variance and $E(c)=0$ ] and the error part of $x$, respectively, with $\operatorname{Cov}(c, u)=0$. Now, if we consider the $p$ vectors of variables $x_{p}$ arranged in a $p \times n$ matrix $X$ and expressed as deviations from the mean $\mu_{p}$, the estimated variance-covariance matrix is

$$
\Sigma_{X}=E\left(X X^{\prime}\right)=D_{\zeta}\left(P_{c}+D_{v}\right) D_{\zeta},
$$

where $\left[P_{c}\right]_{i j}=\operatorname{Cov}\left(c_{i}, c_{j}\right), D_{v}=\operatorname{diag}\left[\operatorname{Cov}\left(u_{i}, u_{i}\right)\right]$, and $D_{\zeta}$ is a diagonal scaling matrix that allows different measurement scales for different manifest variables. The model specifies an equation that precisely defines the mathematical relationship of the angle between common score variables on the circle with the correlation coefficient among common score variables. A correlation structure yielding the required circumplex pattern will be assumed for the common score correlation matrix $P_{c}$, the elements of which are defined by the Fourier series (FS) correlation function

$$
\left[P_{c}\right]_{i j}=\rho\left(\theta_{\mathrm{d}}\right)=\beta_{0}+\sum_{k=1}^{m} \beta_{k} \cos \left(k \theta_{\mathrm{d}}\right),
$$

where $\theta_{\mathrm{d}}=\left(\theta_{j}-\theta_{i}\right)$-that is, the difference of angular position (in radians) on the circumference between two variables. There are certain practical limitations to how many free parameters, $\beta_{k}$, can be specified. At some point, little improvement in the fit of the correlation function will be gained from additional parameters. Additionally, if too many parameters are added, the model may become overparameterized, preventing a proper estimate of the model parameters. Moreover, given that not all of the possible correlation functions that could be fitted to the data will be conceptually sensible within the context of a circular representation, the model places certain logical constraints on the correlation function. For a more detailed discussion of these issues, see Browne (1992, pp. 477, 485-486) and Fabrigar et al. (1997, pp. 191-193). This model has $m$ free parameters for the correlation function, $p-1$ free polar angles $\theta_{i}, p$ free unique variances $v_{i i}$, and $p$ free scaling parameters $\xi_{i i}$, yielding $q=3 p+m-1$ free parameters.

The key assumption of the model is that the circumplex structure of correlations holds for the correlations among common scores, $c$, rather than observed scores, $x$. Thus, the model postulates that even when correlations among common scores have a circumplex structure, correlations among the observed scores should not strictly conform to a circumplex structure; indeed, the structure for the observed scores should approximate circumplex structure (i.e., be quasi-circumplex). In fact, the observed scores contain a unique part, $u$, that is likely to be due in part to 
measurement error, presumably distorting the true structure of the intercorrelations.

\section{Statistical Aspects Considered for the Construction of the Package CircE}

As mentioned above, CircE is a free package written for the $\mathrm{R}$ environment. The $\mathrm{R}$ statistical computing project ${ }^{3}$ is a useful interactive package for data analysis. In comparison with most other statistical packages, $\mathrm{R}$ has at least three compelling advantages: It is free, ${ }^{4}$ runs on multiple platforms, and combines many of the most useful statistical programs into one integrated program. The program itself and detailed installation instructions and manuals are available through CRAN (the Comprehensive R Archive Network). The computational capabilities of $\mathrm{R}$ are extended through user-submitted packages that allow for specialized statistical techniques, graphical devices, and programming interfaces, as well as import/export capabilities to many external data formats. The CircE package is an $\mathrm{R}$ extension useful for researchers who work in the field of personality psychology, and more specifically work with questionnaires subsuming a circumplex latent structure.

The model defined by Equations 2 and 3 is fitted to a sample correlation matrix, $R$, using the maximum likelihood estimation method, which relies on the minimization of the discrepancy function

$$
F(\gamma)=\ln |\Sigma(\gamma)|-\ln |R|+\operatorname{tr}\left[R \Sigma^{-1}(\gamma)\right]-p
$$

over the parameter vector

$$
\gamma=\left[\zeta_{11}, \ldots, \zeta_{p p}, v_{11}, \ldots, v_{p p}, \theta_{1}, \ldots, \theta_{p}, \beta_{1}, \ldots, \beta_{m}\right] .
$$

Initial estimates for these parameters are provided by the factor analysis method described in Browne (1992, section 6.7) and based on the image factor analysis model (Jöreskog, 1969). An iterative optimization is then carried out by minimizing the value of Equation 4 through analytical derivatives (see the supplemental materials: Appendix C, Eqs. 9-20) within the optimization algorithm L-BFGS-B (Byrd, Lu, Nocedal, \& Zhu, 1995; Liu \& Nocedal, 1989; Zhu, Byrd, Lu, \& Nocedal, 1994). The iteration will stop when

$$
\begin{aligned}
& \left(F_{k}-F_{k+1}\right) / \max \left(\left|F_{k}\right|,\left|F_{k+1}\right|, 1\right) \leq \\
& \quad \text { factr } * . \text { Machine\$double.eps, }
\end{aligned}
$$

where $F_{k}$ is the value of Equation 4 at the $k$ th iteration, factr is a variable representing tolerance in the termination test for the algorithm to be set by the user, and .Machinesdouble.eps is the machine precision. The built-in stopping test in Equation 6 is controlled by the parameter factr. It is designed to terminate the run when the change in the objective function $F$ is sufficiently small. The default is factr $=10^{9}$; convergence occurs when the reduction in the objective function is within this factor of the machine tolerance (i.e., a tolerance of about $10^{9} * 2 \times 10^{-16}=2 \times 10^{-7}$ ). The test can be made more stringent by decreasing factr (factr $=$ $10^{12}$ would be low accuracy; factr $=10^{6}$, moderate accuracy; factr $=10^{1}$, extremely high accuracy) and can be nearly disabled by setting factr $=0$ (i.e., the test will stop the algorithm only if the objective func- tion remains unchanged after one iteration). ${ }^{5}$ The code for method L-BFGS-B is written in FORTRAN 77 code obtained from Netlib ${ }^{6}$ (file opt/lbfgs_bcm. shar, or another version is in toms/778) and is inserted in the base distribution of the $\mathrm{R}$ software. A common-score variable is chosen as a reference variable, and its angular position is fixed at $0^{\circ}$. The locations of the other common-score variables on the circle's circumference are specified by polar angles from this reference variable. The polar angle for a target common-score variable is defined as the angle subtended by the vectors joining the center of the circle with the reference common-score variable and the target common-score variable. The standard errors of the parameters are obtained through the inverse of the approximate Hessian matrix, $H$ (see the supplemental materials: Appendix C, Eq. 22). These are used for imposing symmetric confidence intervals on the polar angles $\theta$. The nonsymmetric confidence intervals for the communality index estimates, $\rho\left(x_{i}, c_{i}\right)$ (Browne, 1992, Eq. 4), are obtained from symmetric confidence intervals on $\ln v_{i i}$ (Browne, 1982, pp. 95-96). As previously noted, a correlation function should satisfy certain logical requirements (Browne, 1992, section 5.2); particularly, in Equation 3, $\rho(0)=1$. To ensure that this requirement is satisfied, the equality constraint

$$
\sum_{k=0}^{m} \beta_{k}=1
$$

must be imposed; during the optimization process, parameters $\alpha_{k}$ (denoted by parameters a in the program output in Appendix A) are used to construct the parameters $\beta_{k}$ by means of $\beta_{k}=\alpha_{k} / \sum_{k=0}^{m} \alpha_{k}$. Consequently, the equality constraint in Equation 7 holds. ${ }^{7}$ Another logical requirement for a correlation function is that $\rho_{180^{\circ}} \geq-1$; if the inequality constraints

$$
\beta_{k} \geq 0, k=0,1, \ldots, m
$$

are imposed, this requirement is satisfied (see Browne, 1992, Eqs. 32 and 33). To ensure that values of $\beta_{k}\left(\alpha_{k}\right.$ during the optimization process) are nonnegative, we make use of the optimization method L-BFGS-B, which allows box constraints. That is, each parameter can be given a lower and/or an upper bound. In the same manner, the optimization for parameters $v_{i i}$ is constrained to values $v_{i i} \geq 0$, since $0 \leq \rho\left(x_{i}, c_{i}\right) \leq 1$. Evaluation of the model fit is based on the conventional likelihood ratio (LR) $\chi^{2}$ test and a variety of descriptive measures of the model fit to the sample data (see the output in Appendix A). Given the selection of the discrepancy function in Equation 4, parameter estimation is then carried out by determining the vector of parameter estimates (Equation 5) that produces an estimated covariance matrix as in Equation 2, which in turn yields the minimum value of the discrepancy function, a sample statistic designated here as $\hat{F}$. To evaluate the relative magnitude of $\hat{F}$ so as to achieve an assessment of model fit, the LR $\chi^{2}$ test $(n-1) \hat{F}$ is used under the null hypothesis that the predicted matrix (Equation 2) has the specified model structure, with degrees of freedom $d=p(p+1) / 2-q$, where $q$ is the effective number of parameters. In interpreting the outcome of this test, it 
Table 1

Vocational Interest Scales: Correlation Matrix $(N=175)$

\begin{tabular}{llllllll}
\hline Health & 1.000 & & & & & & \\
Science & 0.654 & 1.000 & & & & & \\
Technology & 0.453 & 0.644 & 1.000 & & & & \\
Trades & 0.251 & 0.440 & 0.757 & 1.000 & & & \\
Business operations & 0.122 & 0.158 & 0.551 & 0.493 & 1.000 & & \\
Business contact & 0.218 & 0.210 & 0.570 & 0.463 & 0.754 & 1.000 & \\
Social & 0.496 & 0.264 & 0.366 & 0.202 & 0.471 & 0.650 & 1.000 \\
\hline
\end{tabular}

is important to recognize that the hypothesis concerns whether the specified model holds exactly in the population and whether any lack of fit in the sample arises only from sampling error (MacCallum, Browne, \& Sugawara, 1996). This is a stringent null hypothesis, and even if a model represents a fairly close approximation to the real world, the test of exact fit will result in a rejection of the model if $n$ is adequately large, as is necessary in CSM. An alternative fit measure widely used in CSM, the RMSEA (root-mean square error of approximation) $\hat{\epsilon}$, proposed by Steiger and Lind (1980) and reviewed by Browne and Cudeck (1992), is preferred, given that (1) the confidence interval for $\epsilon$, denoted $\hat{\epsilon}_{\mathrm{L}} ; \hat{\epsilon}_{\mathrm{U}}$, can be determined; (2) the conventional LR test of exact fit can be redefined as a test of $H_{0}: \epsilon=0$ and accompanied by a more realistic test of close fit, defined as $H_{0}: \epsilon \leq .05$; and (3) as shown by MacCallum et al., it is possible to conduct a power analysis for model fit tests by stating null and alternative hypotheses in terms of $\epsilon$.

Considering developments in the search for more definite cutoff criteria for fit indices in CSM fit assessment (see, e.g., Hu \& Bentler, 1999), a wider group of commonly used fit indices than are provided in CIRCUM were included in CircE (viz., CN, or Hoelter's critical $N$; NFI, normed fit index; NNFI, nonnormed fit index, also known as the Tucker-Lewis index or TLI; CFI, Bentler's comparative fit index; SRMR, standardized root-mean square residual; GFI, goodness-of-fit index; AGFI, adjusted goodness-offit index; AIC, Akaike information criterion; CAIC, Bozdogan's consistent AIC; and BIC, Bayesian information criterion). Although several prominent issues remain unresolved (see, e.g., Fan \& Sivo, 2007, and the references therein) that have not been explicitly examined using Browne's CSPMF, there is evidence for different behaviors of the fit indices under different data conditions. Therefore, if a combination of indices indicate adequate fit levels, more confidence can be assigned to the goodness of the model fit.

\section{Illustrative Examples}

We shall consider two examples of use of the CircE package in fitting a circular model to sample correlation matrices. The results illustrate the convergence of CircE's solutions with those obtained using CIRCUM. In the first example, vocational interest scales, we reanalyzed data used by Browne to illustrate the analysis of circumplex structure with his CSPMF. To better clarify how the CircE program can be used in practice, we fitted four different models to answer specific questions concerning circumplex representations of the data. In this case, using only seven observed variables, data were input directly at the keyboard. In the second example (child interpersonal circumplex), the model was fitted to a data set (see the supplemental materials, file SELF5.sav) containing 48 statements originally assumed to have a circumplex structure and stored in an SPSS (SPSS Inc., 1999) data file that was imported using the appropriate R package (thus avoiding manual input of 1,176 correlation values).

Example 1: Vocational interest scales. The CSPMF was fitted to the correlations among seven vocational interest scales proposed in Browne (1992, Table 2) and reported here in Table 1 . The data can be input into $\mathrm{R}$ directly at the keyboard as in Listing 1 .

In this example, the correlation matrix has been entered in lower triangular form. CircE accepts lower triangular, upper triangular, or symmetric correlation matrices. One can assign the names to the variables (as was done here), which will be passed to CircE. The more general unconstrained model is fitted using the following command line:

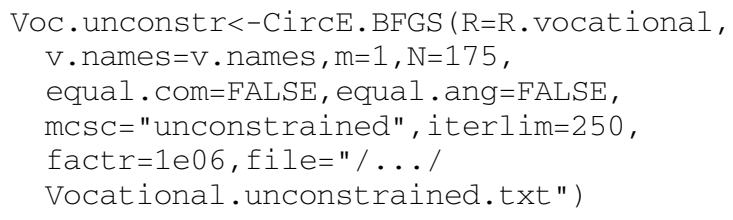

Listing 1

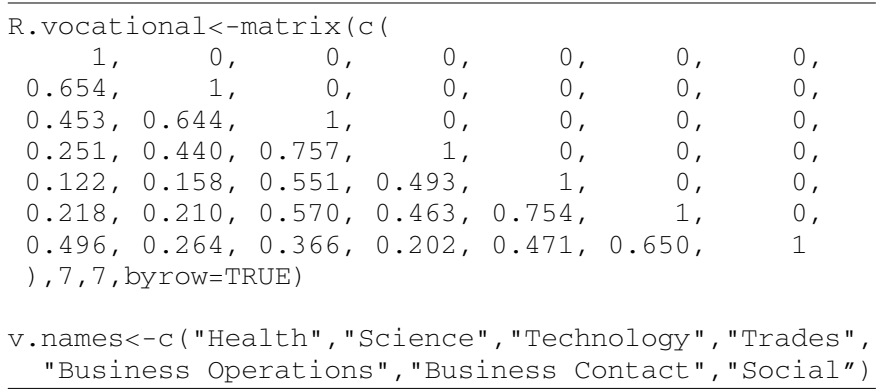




\begin{tabular}{|c|c|c|c|c|}
\hline \\
\hline \multicolumn{5}{|c|}{$\begin{array}{l}\text { names (Voc.unconstr) } \\
{[1] \text { "coeff" }}\end{array}$} \\
\hline [5] & " $\mathrm{PC} "$ & "n " & "q" & "d" \\
\hline [9] & "criterion" & "polar.angles" & "chisq" & "RMSEA " \\
\hline [13] & "RMSEA.U" & "RMSEA.L" & "Fzero" & "Fzero.u" \\
\hline [17] & "Fzero.L" & " chisqNul1" & "dfNul1" & "NFI" \\
\hline$[21]$ & "NNFI " & "CFI" & "residuals" & "standardized.residuals" \\
\hline$[25]$ & " SRMR" & "GFI " & "AGFI " & "CNI " \\
\hline [29] & "BCI " & "AIC" & "CAIC" & "BIC" \\
\hline [33] & "MCSC" & "v.names" & "beta" & "equal. com" \\
\hline$[37]$ & "equal.ang" & " communality" & "communality.index" & "m" \\
\hline
\end{tabular}

where CircE.BFGS () is the function that defines the circumplex estimation via L-BFGS-B optimization, the arguments of which (explained in $\mathrm{R}$ help documentation that may be called via ?CirCE.BFGS) are the following: the correlation matrix label, variable labels, number of free parameters in the correlation function (Equation 3), sample size, two logical arguments specifying whether certain constraints should be placed on variable locations around the circle and/or on the unique variance amount, an option for constraining the MCSC value (mesc $=$ "unconstrained", "0", or "-1"), the maximum number of iterations for the optimization, and the tolerance for the stopping test (Equation 6). CircE returns a printed report (see Appendix A) that can be saved as a text file by specifying an appropriate path with an argument $\mathrm{file}$, as well as returning an object on which to perform additional computations (e.g., producing plots). In our examples, the object named Voc. unconstr contains several attributes that could be extracted and used directly with other R functions, as shown in Listing 2.

As stated above, the unconstrained model could be considered general: In fact, we can obtain nested models that are special cases of the unconstrained one, if we place specific constraints on the parameters. These models can be compared in order to test specific hypotheses about the data using a $\chi^{2}$ difference test (Bollen, 1989). For example, the hypothesis of comparable amounts of unique variance can be tested by specifying a model in which the communality estimates, and more precisely the elements of $\operatorname{diag}\left[D_{v}\right]$ (see Browne, 1992, pp. 471-472), are constrained to be equal. If the fit of the constrained model is not significantly weaker than that of the unconstrained model, the constraint has statistical plausibility (i.e., an equal amount of common variance in each variable). Such a model can be specified by setting the parameter equal. com as true:

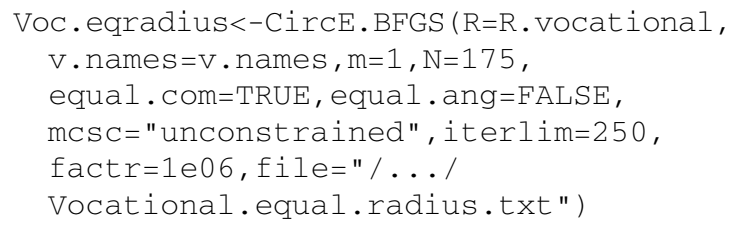

Moreover, a researcher might wish also to test whether the observed variables are equally spaced around the circumference. Such a hypothesis can be tested by specifying a model in which the variable polar angles are equally spaced around $360^{\circ}$. Using the string above, we would only need to set the parameter equal . ang as true:

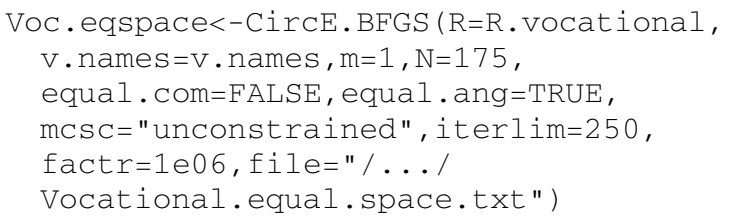

The matrix depicted in Table 1 has nonnegative elements. Clearly, there is a circular ordering of interest scales such that adjacent scales have high correlations. The correlations tend to decrease as the separation between tests increases, but then they increase after an ideal distance of $180^{\circ}$ of separation has been reached. Since the coefficients are positive, the value of $\hat{\rho}_{180^{\circ}}$ could be set to 0 , imposing mcsc $=$ " 0 " (another possible option is $\hat{\rho}_{180^{\circ}}=-1$ for negative correlation coefficients). For the four fitted models, maximum likelihood estimates of polar angles and communality indices, estimated Fourier series weights, and fit measures (sample discrepancy function value $\hat{F}$, population discrepancy function value $\hat{F}_{0}$, and RMSEA index $\hat{\epsilon}$ ) are reported in Tables 2 and 3 .

The results for $m=1, m=2$, and $m=3$ in Equation 3 coincide precisely with the ones obtained by CIRCUM. Particularly, as Browne (1992, p. 494) also reported, the FS correlation function with $m=2$ gave two Heywood cases, $\hat{\rho}_{\left(x_{i}, c_{i}\right)}=1$, in the unconstrained model, and FS with $m=3$ resulted in a correlation function weight estimate, $\hat{\beta}_{3}$, attaining the lower bound of zero.

Example 2: Child interpersonal circumplex. To illustrate how CircE can be used to test the circumplex structure of dozens of variables (e.g., personality questionnaire items), we applied CSPMF estimation to a recently collected data set to examine the structural continuity of the interpersonal circumplex model in childhood (Di Blas, Grassi, Luccio, \& Momentè, 2008). Specifically, we analyzed 286 self-reports provided by 5 th-grade children who rated their interpersonal behavior on 48 items (i.e., six items for each of eight scale octants) conceptually organized around the dominance (DOM) and love (LOV) domains according to Wiggins's interpersonal circumplex (IPC) model (Wiggins, 1979; Wiggins \& Trapnell, 1996). Briefly, Wiggins's model is based on the idea that people who interact attempt to negotiate relations of hierarchy and cooperation by granting or denying the resources of power (dominance) and warmth (love). Accordingly, the IPC model differently combines elements of the reference axes (DOM and LOV) and defines eight possible interpersonal styles circularly ordered in terms of DOM and LOV, in compliance with a law of neighboring, positing that two 
Table 2

Vocational Interest Scales: Estimates

\begin{tabular}{ccccccccccccccccccccc}
\hline Model & $m$ & $\hat{\beta}_{0}$ & $\hat{\beta}_{1}$ & $\hat{\beta}_{2}$ & $\hat{\beta}_{3}$ & $\hat{\rho}_{180^{\circ}}$ & $\hat{\theta}_{1}$ & $\hat{\theta}_{2}$ & $\hat{\theta}_{3}$ & $\hat{\theta}_{4}$ & $\hat{\theta}_{5}$ & $\hat{\theta}_{6}$ & $\hat{\theta}_{7}$ & $\hat{\rho}_{1}$ & $\hat{\rho}_{2}$ & $\hat{\rho}_{3}$ & $\hat{\rho}_{4}$ & $\hat{\rho}_{5}$ & $\hat{\rho}_{6}$ & $\hat{\rho}_{7}$ \\
\hline $1^{\mathrm{a}}$ & 1 & .638 & .362 & & & .28 & 0 & 55 & 112 & 123 & 192 & 210 & 269 & .93 & .81 & .98 & .78 & .80 & .94 & .83 \\
& 2 & .608 & .355 & .038 & & .29 & 0 & 52 & 106 & 117 & 176 & 192 & 263 & .96 & .83 & 1 & .77 & .82 & .94 & 1 \\
& 3 & .608 & .355 & .038 & 0 & .29 & 0 & 52 & 106 & 117 & 176 & 192 & 263 & .96 & .83 & 1 & .77 & .82 & .94 & 1 \\
$2^{\mathrm{b}}$ & 1 & .628 & .372 & & & .26 & 0 & 48 & 111 & 121 & 190 & 208 & 272 & .87 & - & - & - & - & - & - \\
& 2 & .619 & .370 & .011 & & .26 & 0 & 50 & 111 & 122 & 190 & 209 & 272 & .88 & - & - & - & - & - & - \\
$3^{\mathrm{c}}$ & 1 & .704 & .296 & & & .41 & 0 & 51 & 103 & 154 & 206 & 257 & 309 & .81 & .86 & .92 & .81 & .74 & .97 & .80 \\
& 2 & .668 & .297 & .035 & & .41 & 0 & 51 & 103 & 154 & 206 & 257 & 309 & .88 & .87 & .96 & .82 & .75 & 1 & .83 \\
$4^{\mathrm{d}}$ & 1 & .500 & .500 & & & 0 & 0 & 59 & 112 & 122 & 191 & 209 & 263 & 1 & .66 & .97 & .64 & .67 & .91 & .66 \\
& 2 & .500 & .500 & .000 & & 0 & 0 & 59 & 112 & 122 & 191 & 209 & 263 & 1 & .78 & .99 & .76 & .79 & .95 & .78 \\
\hline
\end{tabular}

${ }^{\mathrm{a}}$ Communality indices $\rho_{i}$, polar angles $\theta_{i}$, and minimal common score correlation $\rho_{180^{\circ}}$ are unconstrained. ${ }^{\mathrm{b} C o m m u n a l i t y ~ i n d i c e s ~ a r e ~ c o n-~}$ strained to be equal. $\quad$ CPolar angles are constrained to be equally spaced around $360^{\circ}$. ${ }^{\mathrm{d}}$ Constraint was applied on the minimal common score correlation: $\rho_{180^{\circ}}=0$.

Table 3

Vocational Interest Scales: Fit Measures

\begin{tabular}{cccccccc}
\hline Model & $m$ & $\hat{F}$ & $\hat{F}_{0}$ & $\left(\hat{F}_{0_{\mathrm{L}}} ; \hat{F}_{0_{\mathrm{U}}}\right)$ & $\hat{\epsilon}$ & $\left(\hat{\epsilon}_{\mathrm{L}} ; \hat{\epsilon}_{\mathrm{U}}\right)$ & Heywood $^{\mathrm{a}}$ \\
\hline 1 & 1 & .09 & .049 & $(.005 ; .139)$ & .084 & $(.026 ; .141)$ & 0 \\
& 2 & .067 & .032 & $(0, j .111)$ & .073 & $(.000 ; .136)$ & 2 \\
& 3 & .067 & .038 & $(.001 ; .119)$ & .087 & $(.017 ; .154)$ & 2 \\
2 & 1 & .299 & .223 & $(.117 ; .376)$ & .131 & $(.095 ; .170)$ & 0 \\
& 2 & .292 & .222 & $(.118 ; .372)$ & .136 & $(.099 ; .176)$ & 0 \\
3 & 1 & .574 & .499 & $(.337 ; .706)$ & .196 & $(.161 ; .233)$ & 0 \\
& 2 & .521 & .452 & $(.299 ; .648)$ & .194 & $(.158 ; .232)$ & 1 \\
4 & 1 & .174 & .127 & $(.052 ; .248)$ & .126 & $(.081 ; .176)$ & 1 \\
& 2 & .174 & .133 & $(.057 ; .253)$ & .138 & $(.090 ; .190)$ & 1 \\
\hline${ }^{\mathrm{a}} \hat{\rho}_{\left(x_{i}, c_{i}\right)}=1$. & & & & & & \\
\end{tabular}

variables are neighbors if they share more variance with each other than with other variables (see Figure 1).

Using the modified version of the CIRCUM program for large correlation matrices, the circular structure of the interpersonal items was analyzed by means of a confirmatory approach, which yielded the unconstrained (model) estimates presented in Table 4B. The confirmatory analysis was exactly replicated using the CircE package, and

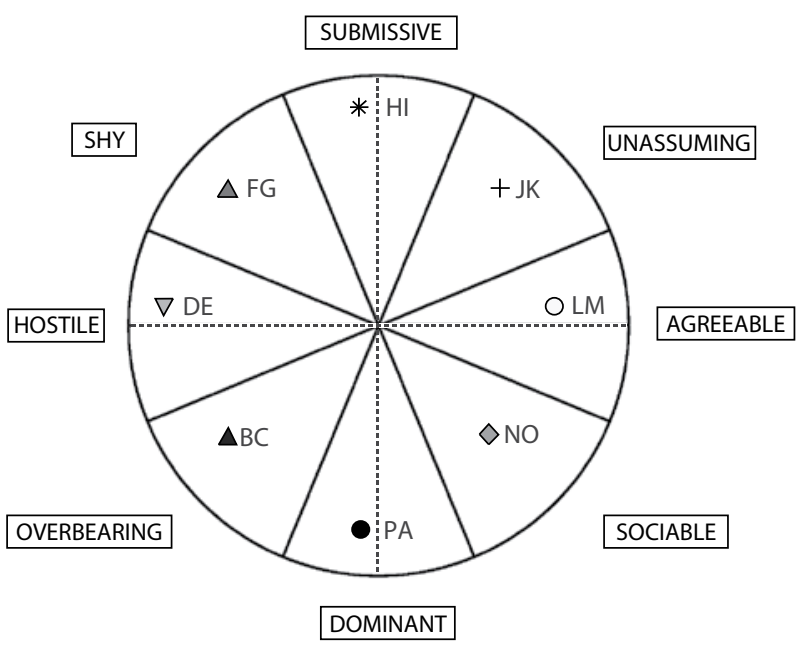

Figure 1. IPC model. The interpersonal space is divided into eight circularly ordered categories (octants): LM-Agreeable, NOSociable, PA-Dominant, BC-Overbearing, DE-Hostile, FG-Shy, HI-Submissive, and JK-Unassuming. The labels LM, NO, PA, BC, DE, FG, HI, and JK are conventionally used in the interpersonal circumplex model (see Wiggins, 1979). the results are reported in Table 4A. Data again were initially input using the SPSS statistical program, and a sample correlation matrix, $R$, was obtained directly by the software. To run the external program CIRCUM, the user must provide an input text file, which contains several settings similar to those required as arguments by the CircE functions presented in the previous example. To fit the circumplex model to the items of the interpersonal questionnaire, the correlation matrix for the 48 items must be inserted manually into the input text file, a time-consuming operation that also increases the probability of error in data insertion. The possibility of directly using a CSPMF estimation utility within the same software used for data entry is the first practical advantage of the CircE package. Since the data and the CSPMF routine are integrated in the same statistical program, it is no longer necessary to prepare an external input text file.

As a first step, data could be easily imported into the $\mathrm{R}$ environment using the function read.spss of the package "foreign" (R Core Team Members, DebRoy, Bivand, et al., 2008), depicted in Listing 3. Then, supposing that the column of the original file contains our 48 items, we can easily compute the sample correlation matrix among them. As a second step, the CircE package can be loaded, to directly estimate an unconstrained CSPMF model:

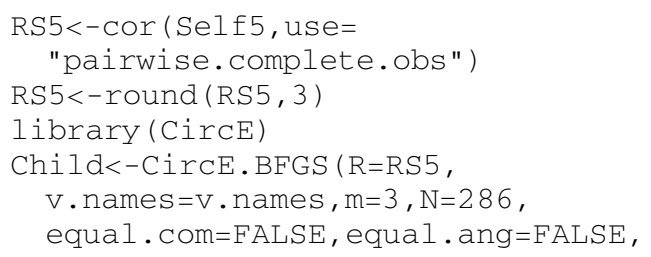


Listing 3

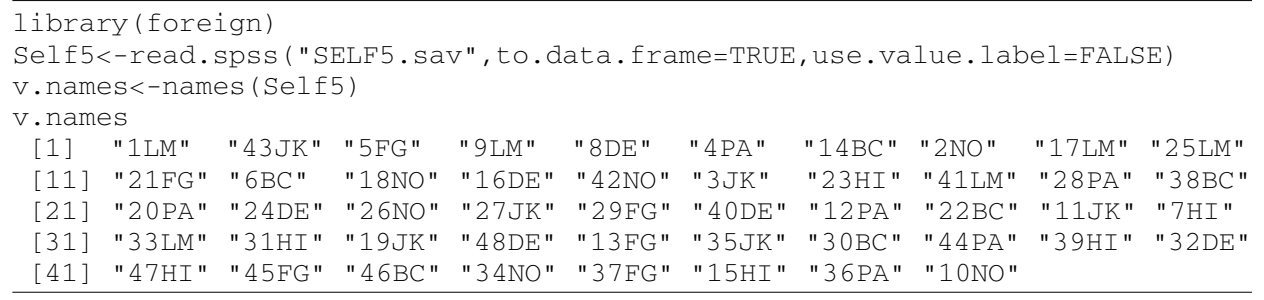

mcsc $=$ "unconstrained" , iterlim=250, factr $=1 \mathrm{e} 10$ )

As anticipated, another notable practical advantage is the possibility to produce a graphical representation of the estimated circular order using the function CirCE.PIot () (see Figure 2).

To simplify the assignment of a color and character type to each point on the graph — both of which are required as arguments by the function CirCE.Plot () - in the case of a large number of items, the function char.assign ( ) can be used as follows:

- Supposing that the items are tagged with reference to the relative scale (e.g., Table 4A, first column), it is sufficient to create a string with scale names (sc.names),

sc.names=c ( "LM" , "NO" , "PA" , "BC", "DE" , "FG" , "HI ", " JK" )

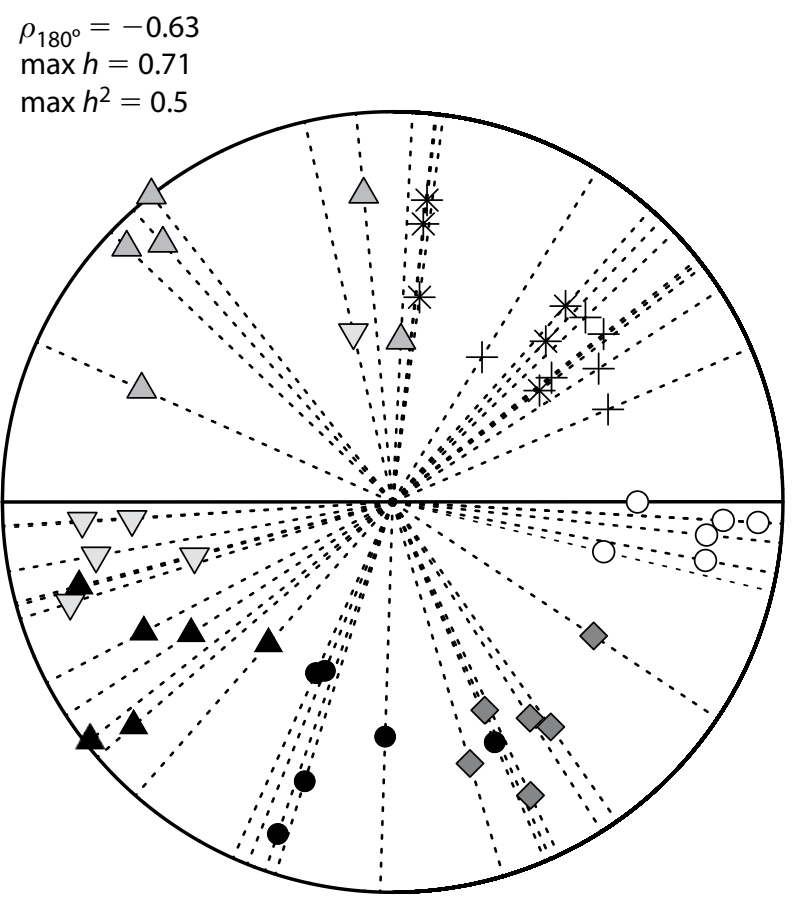

Attended Circular Order

$\bigcirc \diamond \bullet \triangle \nabla \triangle *+$

LM NO PA BC DE FG HI JK
- a vector of the same length containing the point characters (point. char),

point. char $=\mathrm{C}(21,23,16,17,25,24,8,3)$

- and a vector of color names for filling in the points (bg.point).

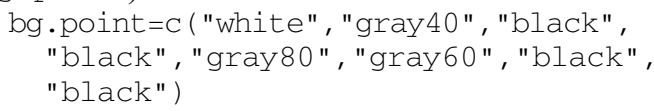

The char.assign ( ) function matches scale names with the item names (the vector $v$. names) and assigns appropriate characters and colors automatically. The result is an R object (A) that contains two vectors of the same length, shown in Listing 4, of variable names that will be passed to CircE.Plot ( ).

Octant-bounded estimation. For the previous example, child interpersonal circumplex, quantitative modelfitting indices (Table 4A, first row) showed that the 5th

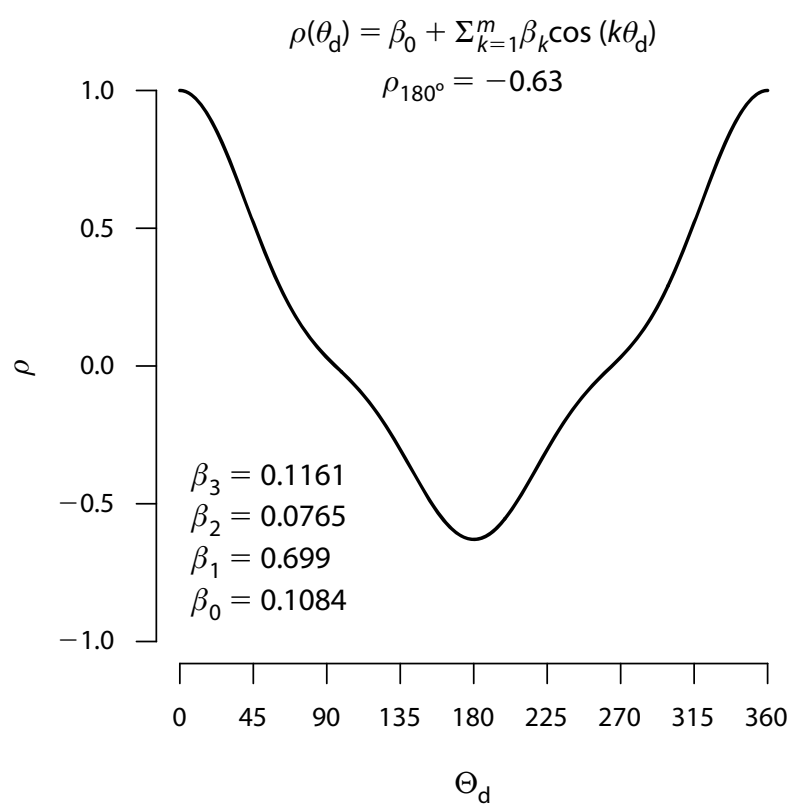

Figure 2. Child interpersonal circumplex: Unconstrained circular ordering of the 48 items, obtained using the CirCE . Plot function. 
Table 4

Estimates of Unknown Parameters: Comparison Between the Two Programs

\begin{tabular}{|c|c|c|c|c|c|c|c|c|c|}
\hline \multicolumn{5}{|c|}{ (A) $\operatorname{CircE}$} & \multicolumn{5}{|c|}{ (B) CIRCUM } \\
\hline$\hat{F}$ & $\hat{F}_{0}$ & $\left(\hat{F}_{0_{\mathrm{L}}} ; \hat{F}_{0_{\mathrm{U}}}\right)$ & $\hat{\epsilon}$ & $\left(\hat{\epsilon}_{\mathrm{L}} ; \hat{\epsilon}_{\mathrm{U}}\right)$ & $\hat{F}$ & $\hat{F}_{0}$ & $\left(\hat{F}_{0_{\mathrm{L}}} ; \hat{F}_{0_{\mathrm{U}}}\right)$ & $\hat{\epsilon}$ & $\left(\hat{\epsilon}_{\mathrm{L}} ; \hat{\epsilon}_{\mathrm{U}}\right)$ \\
\hline \multirow[t]{2}{*}{5.86} & 2.27 & $(1.90 ; 2.68)$ & .047 & $(.043 ; .051)$ & 5.86 & 2.25 & $(1.87 ; 2.65)$ & .047 & $(.043 ; .051)$ \\
\hline & $\hat{\theta}_{i}$ & $\left(\hat{\theta}_{i_{\mathrm{L}}} ; \hat{\theta}_{i_{\mathrm{U}}}\right)$ & $\hat{\rho}_{(x, c)}$ & $\left(\hat{\rho}_{\mathrm{L}} ; \hat{\rho}_{\mathrm{U}}\right)$ & & $\hat{\theta}_{i}$ & $\left(\hat{\theta}_{i_{\mathrm{L}}} ; \hat{\theta}_{i_{\mathrm{U}}}\right)$ & $\hat{\rho}_{(x, c)}$ & $\left(\hat{\rho}_{\mathrm{L}} ; \hat{\rho}_{\mathrm{U}}\right)$ \\
\hline $1 \mathrm{LM}$ & 0 & $(0 ; 0)$ & .44 & $(.34 ; .56)$ & $1 \mathrm{LM}$ & 0 & $(0 ; 0)$ & .45 & $(.34 ; .56)$ \\
\hline $27 \mathrm{JK}$ & 23 & $(5 ; 41)$ & .43 & $(.32 ; .55)$ & $27 \mathrm{JK}$ & 23 & $(5 ; 41)$ & .43 & $(.33 ; .55)$ \\
\hline $3 \mathrm{JK}$ & 33 & $(15 ; 51)$ & .45 & $(.34 ; .56)$ & $3 \mathrm{JK}$ & 33 & $(15 ; 50)$ & .45 & $(.35 ; .57)$ \\
\hline $31 \mathrm{HI}$ & 37 & $(16 ; 59)$ & .33 & $(.23 ; .47)$ & $31 \mathrm{HI}$ & 37 & $(16 ; 58)$ & .34 & $(.24 ; .48)$ \\
\hline $43 \mathrm{JK}$ & 38 & $(18 ; 58)$ & .37 & $(.26 ; .50)$ & $43 \mathrm{JK}$ & 38 & $(18 ; 57)$ & .38 & $(.27 ; .51)$ \\
\hline $35 \mathrm{JK}$ & 39 & $(21 ; 56)$ & .49 & $(.39 ; .60)$ & $35 \mathrm{JK}$ & 38 & $(21 ; 55)$ & .48 & $(.38 ; .60)$ \\
\hline $11 \mathrm{JK}$ & 44 & $(26 ; 61)$ & .48 & $(.38 ; .60)$ & $11 \mathrm{JK}$ & 44 & $(26 ; 61)$ & .48 & $(.38 ; .60)$ \\
\hline $15 \mathrm{HI}$ & 46 & $(27 ; 66)$ & .40 & $(.30 ; .53)$ & $15 \mathrm{HI}$ & 46 & $(27 ; 65)$ & .42 & $(.31 ; .54)$ \\
\hline 39HI & 49 & $(31 ; 66)$ & .47 & $(.37 ; .59)$ & $39 \mathrm{HI}$ & 48 & $(30 ; 65)$ & .48 & $(.37 ; .59)$ \\
\hline 19JK & 58 & $(35 ; 82)$ & .31 & $(.20 ; .45)$ & $19 J K$ & 57 & $(33 ; 82)$ & .29 & $(.18 ; .44)$ \\
\hline $47 \mathrm{HI}$ & 83 & $(62 ; 103)$ & .37 & $(.27 ; .51)$ & $47 \mathrm{HI}$ & 83 & $(62 ; 103)$ & .38 & $(.27 ; .51)$ \\
\hline 23HI & 83 & $(67 ; 100)$ & .55 & $(.45 ; .66)$ & $23 \mathrm{HI}$ & 83 & $(67 ; 100)$ & .56 & $(.46 ; .67)$ \\
\hline $7 \mathrm{HI}$ & 84 & $(66 ; 101)$ & .51 & $(.40 ; .62)$ & $7 \mathrm{HI}$ & 83 & $(66 ; 101)$ & .51 & $(.41 ; .63)$ \\
\hline $29 \mathrm{FG}$ & 87 & $(63 ; 111)$ & .29 & $(.19 ; .44)$ & $29 \mathrm{FG}$ & 87 & $(61 ; 112)$ & .26 & $(.16 ; .42)$ \\
\hline $21 \mathrm{FG}$ & 95 & $(79 ; 112)$ & .56 & $(.46 ; .67)$ & $21 \mathrm{FG}$ & 95 & $(78 ; 112)$ & .56 & $(.46 ; .66)$ \\
\hline 24DE & 103 & $(80 ; 126)$ & .32 & $(.21 ; .46)$ & 24DE & 103 & $(80 ; 125)$ & .32 & $(.21 ; .46)$ \\
\hline $13 \mathrm{FG}$ & 128 & $(112 ; 144)$ & .71 & $(.62 ; .79)$ & $13 \mathrm{FG}$ & 128 & $(112 ; 144)$ & .71 & $(.62 ; .79)$ \\
\hline $5 \mathrm{FG}$ & 131 & $(115 ; 148)$ & .63 & $(.54 ; .72)$ & $5 \mathrm{FG}$ & 131 & $(115 ; 148)$ & .63 & $(.54 ; .72)$ \\
\hline 37FG & 136 & $(120 ; 152)$ & .67 & $(.58 ; .75)$ & $37 \mathrm{FG}$ & 136 & $(120 ; 152)$ & .67 & $(.58 ; .75)$ \\
\hline $45 \mathrm{FG}$ & 156 & $(139 ; 173)$ & .50 & $(.40 ; .61)$ & $45 \mathrm{FG}$ & 155 & $(138 ; 172)$ & .50 & $(.39 ; .61)$ \\
\hline $48 \mathrm{DE}$ & 183 & $(167 ; 199)$ & .56 & $(.47 ; .66)$ & $48 \mathrm{DE}$ & 183 & $(167 ; 200)$ & .56 & $(.47 ; .66)$ \\
\hline $16 \mathrm{DE}$ & 184 & $(166 ; 201)$ & .47 & $(.37 ; .58)$ & $16 \mathrm{DE}$ & 184 & $(166 ; 201)$ & .47 & $(.37 ; .58)$ \\
\hline $8 \mathrm{DE}$ & 190 & $(174 ; 207)$ & .55 & $(.45 ; .64)$ & $8 \mathrm{DE}$ & 190 & $(173 ; 206)$ & .55 & $(.46 ; .65)$ \\
\hline $38 \mathrm{BC}$ & 195 & $(179 ; 211)$ & .59 & $(.50 ; .68)$ & $38 \mathrm{BC}$ & 195 & $(179 ; 211)$ & .59 & $(.50 ; .68)$ \\
\hline 40DE & 195 & $(175 ; 216)$ & .37 & $(.27 ; .50)$ & $40 \mathrm{DE}$ & 195 & $(175 ; 216)$ & .38 & $(.28 ; .50)$ \\
\hline $32 \mathrm{DE}$ & 197 & $(181 ; 213)$ & .61 & $(.53 ; .70)$ & $32 \mathrm{DE}$ & 197 & $(181 ; 213)$ & .61 & $(.53 ; .70)$ \\
\hline $14 \mathrm{BC}$ & 208 & $(190 ; 225)$ & .51 & $(.41 ; .61)$ & $14 \mathrm{BC}$ & 207 & $(190 ; 224)$ & .51 & $(.41 ; .61)$ \\
\hline $22 \mathrm{BC}$ & 213 & $(194 ; 232)$ & .44 & $(.34 ; .55)$ & $22 \mathrm{BC}$ & 213 & $(194 ; 232)$ & .43 & $(.33 ; .54)$ \\
\hline $46 \mathrm{BC}$ & 218 & $(203 ; 234)$ & .70 & $(.62 ; .77)$ & $46 \mathrm{BC}$ & 218 & $(203 ; 233)$ & .70 & $(.62 ; .77)$ \\
\hline $6 \mathrm{BC}$ & 221 & $(205 ; 237)$ & .62 & $(.53 ; .71)$ & $6 \mathrm{BC}$ & 221 & $(205 ; 236)$ & .62 & $(.54 ; .71)$ \\
\hline $30 \mathrm{BC}$ & 229 & $(207 ; 250)$ & .34 & $(.24 ; .47)$ & $30 \mathrm{BC}$ & 229 & $(207 ; 250)$ & .35 & $(.25 ; .48)$ \\
\hline 20PA & 246 & $(224 ; 267)$ & .34 & $(.23 ; .48)$ & $20 \mathrm{PA}$ & 246 & $(225 ; 267)$ & .35 & $(.24 ; .48)$ \\
\hline 4PA & 248 & $(226 ; 270)$ & .33 & $(.22 ; .47)$ & $4 \mathrm{PA}$ & 249 & $(227 ; 270)$ & .33 & $(.22 ; .47)$ \\
\hline 36PA & 251 & $(235 ; 267)$ & .64 & $(.54 ; .73)$ & $36 \mathrm{PA}$ & 251 & $(235 ; 266)$ & .64 & $(.54 ; .73)$ \\
\hline 44PA & 252 & $(235 ; 269)$ & .53 & $(.43 ; .64)$ & 44PA & 252 & $(235 ; 269)$ & .53 & $(.42 ; .63)$ \\
\hline $12 \mathrm{PA}$ & 268 & $(249 ; 287)$ & .43 & $(.32 ; .55)$ & $12 \mathrm{PA}$ & 268 & $(249 ; 287)$ & .42 & $(.31 ; .54)$ \\
\hline $18 \mathrm{NO}$ & 286 & $(268 ; 304)$ & .49 & $(.39 ; .61)$ & $18 \mathrm{NO}$ & 286 & $(268 ; 304)$ & .49 & $(.39 ; .60)$ \\
\hline 28PA & 293 & $(275 ; 311)$ & .47 & $(.37 ; .59)$ & $28 \mathrm{PA}$ & 292 & $(274 ; 311)$ & .47 & $(.36 ; .58)$ \\
\hline $42 \mathrm{NO}$ & 294 & $(274 ; 314)$ & .41 & $(.31 ; .54)$ & $42 \mathrm{NO}$ & 293 & $(274 ; 313)$ & .42 & $(.31 ; .54)$ \\
\hline $34 \mathrm{NO}$ & 295 & $(279 ; 312)$ & .59 & $(.49 ; .69)$ & $34 \mathrm{NO}$ & 295 & $(278 ; 311)$ & .59 & $(.49 ; .69)$ \\
\hline $26 \mathrm{NO}$ & 303 & $(284 ; 321)$ & .46 & $(.36 ; .58)$ & $26 \mathrm{NO}$ & 302 & $(283 ; 320)$ & .46 & $(.36 ; .58)$ \\
\hline $2 \mathrm{NO}$ & 305 & $(288 ; 323)$ & .50 & $(.39 ; .61)$ & $2 \mathrm{NO}$ & 304 & $(287 ; 322)$ & .50 & $(.40 ; .61)$ \\
\hline $10 \mathrm{NO}$ & 326 & $(308 ; 344)$ & .44 & $(.33 ; .56)$ & $10 \mathrm{NO}$ & 325 & $(307 ; 343)$ & .43 & $(.33 ; .56)$ \\
\hline $33 \mathrm{LM}$ & 347 & $(328 ; 6)$ & .39 & $(.29 ; .52)$ & $33 \mathrm{LM}$ & 346 & $(328 ; 5)$ & .41 & $(.31 ; .53)$ \\
\hline 41LM & 349 & $(334 ; 5)$ & .58 & $(.48 ; .67)$ & $41 \mathrm{LM}$ & 349 & $(334 ; 4)$ & .58 & $(.48 ; .67)$ \\
\hline $17 \mathrm{LM}$ & 354 & $(339 ; 9)$ & .57 & $(.48 ; .67)$ & $17 \mathrm{LM}$ & 354 & $(338 ; 9)$ & .57 & $(.48 ; .67)$ \\
\hline 9LM & 357 & $(342 ; 12)$ & .60 & $(.51 ; .69)$ & 9LM & 356 & $(341 ; 11)$ & .60 & $(.51 ; .69)$ \\
\hline $25 \mathrm{LM}$ & 357 & $(342 ; 11)$ & .66 & $(.58 ; .74)$ & $25 \mathrm{LM}$ & 356 & $(342 ; 11)$ & .67 & $(.58 ; .75)$ \\
\hline \multicolumn{5}{|c|}{ CPU time $36 \mathrm{~min}$} & \multicolumn{5}{|c|}{ CPU time $27 \mathrm{~min}$} \\
\hline
\end{tabular}

graders' ratings conformed to a circumplex structure; that is, the 48 sentences could be represented in a circular array. Nevertheless, to develop a psychological measure, we need to demonstrate that each item represents the intended variable, in agreement with the psychological model, in order to provide evidence of the measure's structural validity. To help with this aim, we may inspect the item continuum illustrated in Figure 2. Overall, the observed sentence array seems to reproduce fairly well the hypothesized circular array shown in Figure 1. A closer inspection, however, reveals that some sentences intended to represent different interpersonal variables overlap (e.g., JK and HI sentences) and that items representing the same interpersonal variable are not regularly distributed within $45^{\circ}$ arcs, as the ICM posits (e.g., FG items are spread through the upper left quadrant, but $\mathrm{DE}, \mathrm{BC}$, and $\mathrm{PA}$ sentences are all arranged within the lower left quadrant). Therefore, whatever decision we make about the structural validity of our 48-item measure, such a decision would be based on personal judgments. Conversely, we primarily need to test whether each item represents what it is intended to represent, and thereby whether it falls within an expected $\left(45^{\circ}\right)$ arc. 
Listing 4

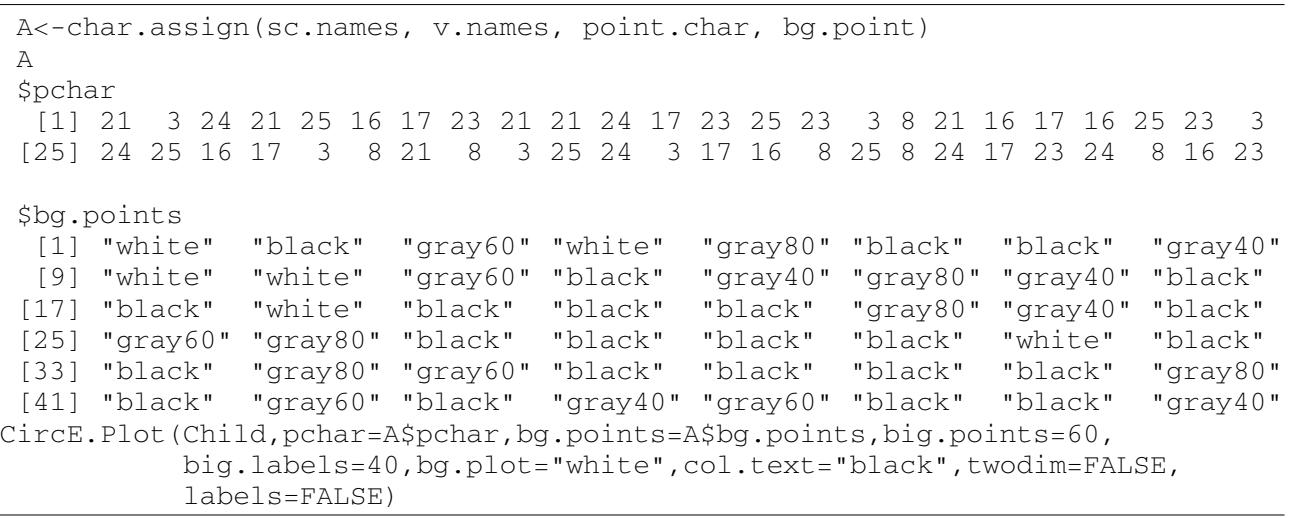

The models that can be tested with CIRCUM do not serve this purpose. Thus, we developed a function aimed at testing a model in which items are constrained to fall within a given arc, but within that arc, each item's angular position and radius are unconstrained. The function CirCE. BFGS has two arguments, upper and lower, which specify, respectively, the upper and lower bounds for each item, or variable, used for a bounded optimization; a bound assignment functionality, bound. assign (), helps provide each of the 48 items with an upper and lower bound, as seen previously for char.assign(). The code in R for fitting the octant-bounded model is:
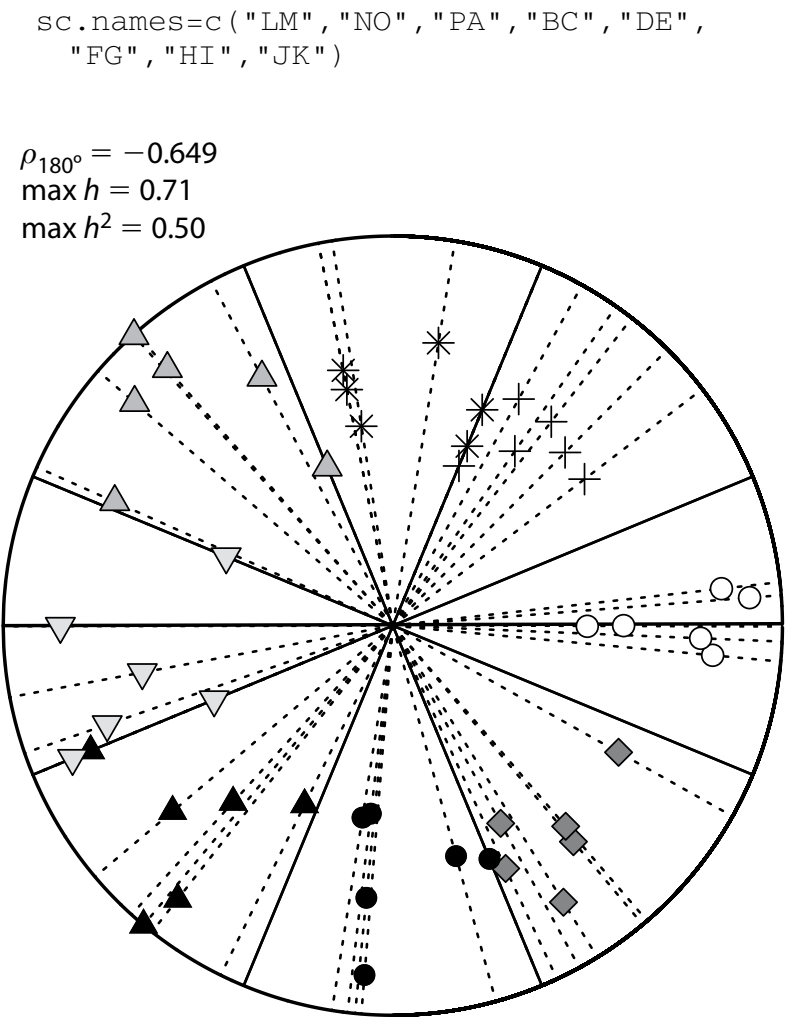

Attended Circular Order

$\bigcirc \diamond \diamond \triangle \nabla \triangle *+$

LM NO PA BC DE FG HI JK lower $<-c(-22.5,292.5,247.5,202.5,157.5$ $112.5,67.5,22.5)$

upper $<-c(22.5,337.5,292.5,247.5,202.5$, $157.5,112.5,67.5)$

$\mathrm{B}<-$ bound.assign ( $\mathrm{sc}$. names, $\mathrm{V}$. names, lower, upper)

Child.obm<-CircE. BFGS ( $R=$ RS 5 ,

$\mathrm{V} \cdot$ names $=\mathrm{V}$. names $, \mathrm{m}=3, \mathrm{~N}=286$,

equal . com=FALSE, equal . ang=FALSE, mcsc $=$ "unconstrained" , iterlim $=250$,

factr=1e10, upper=B $\$$ upper, lower=B lower $)$

The estimated octant-bounded angular position can be graphically represented (see Figure 3 ) using the function CircE.Plot (), as in the previous example.

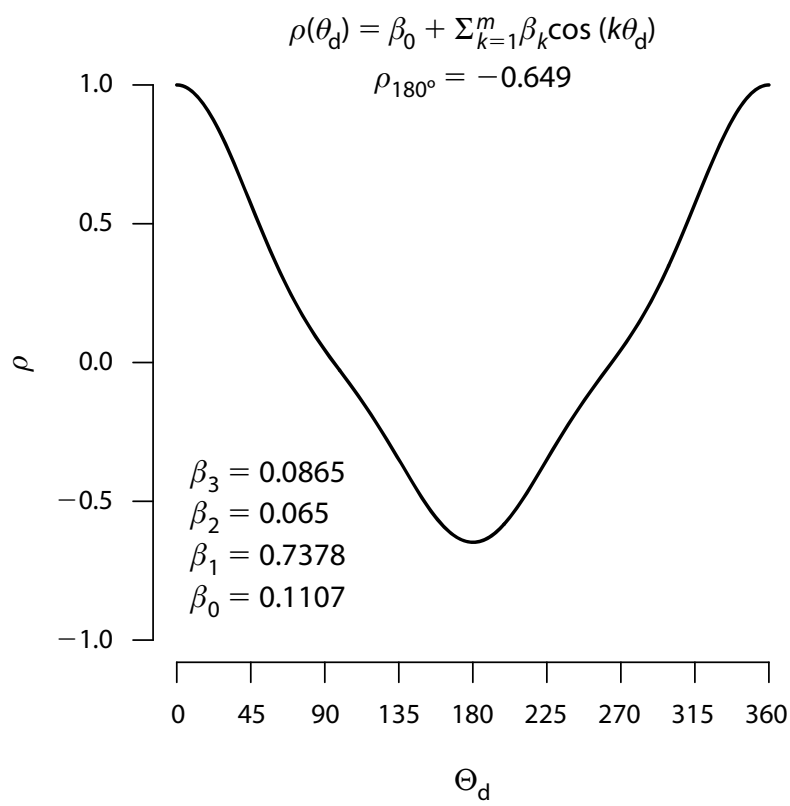

Figure 3. Child interpersonal circumplex: Octant-bounded circular ordering of the 48 items. 
Comparing the solution obtained by putting no constraints on the model with the one observed when constraining each item to fall within a specific arc (Table 5) reveals that this specific constraint affects only the angular locations of items, $\hat{\theta}_{i}$, not the communality indices, $\hat{\rho}_{(x, c)}$. Moreover, changes in $\hat{\rho}_{(x, c)}$ are linearly independent from changes in $\hat{\theta}_{i}$ (Pearson's $r=.0781$ ). Testing an octant-bounded model revealed that our 48 items were in agreement with the hypothesized psychological model of interpersonal qualities, with $\hat{F}=5.928$ and $\hat{\epsilon}=.047$ [90\% confidence interval $=$
$(.043, .051)]$. These results are informative not only about the circularity of the item array, but also about the tenability of a specific circular item organization.

We have tested the CSPMF on the correlation matrix with items measured to 3 decimal positions. Table 6 shows that retaining up to 12 decimal positions and using the stopping test in Equation 6 more stringently, with factr $=$ $10^{6}$ and iterlim $=1,000$, does not produce substantial changes in the results of the unconstrained estimates, nor in the octant-bounded model.

Table 5

Estimates of Unknown Parameters: Comparison Between Unconstrained Model and Octant-Bounded Model

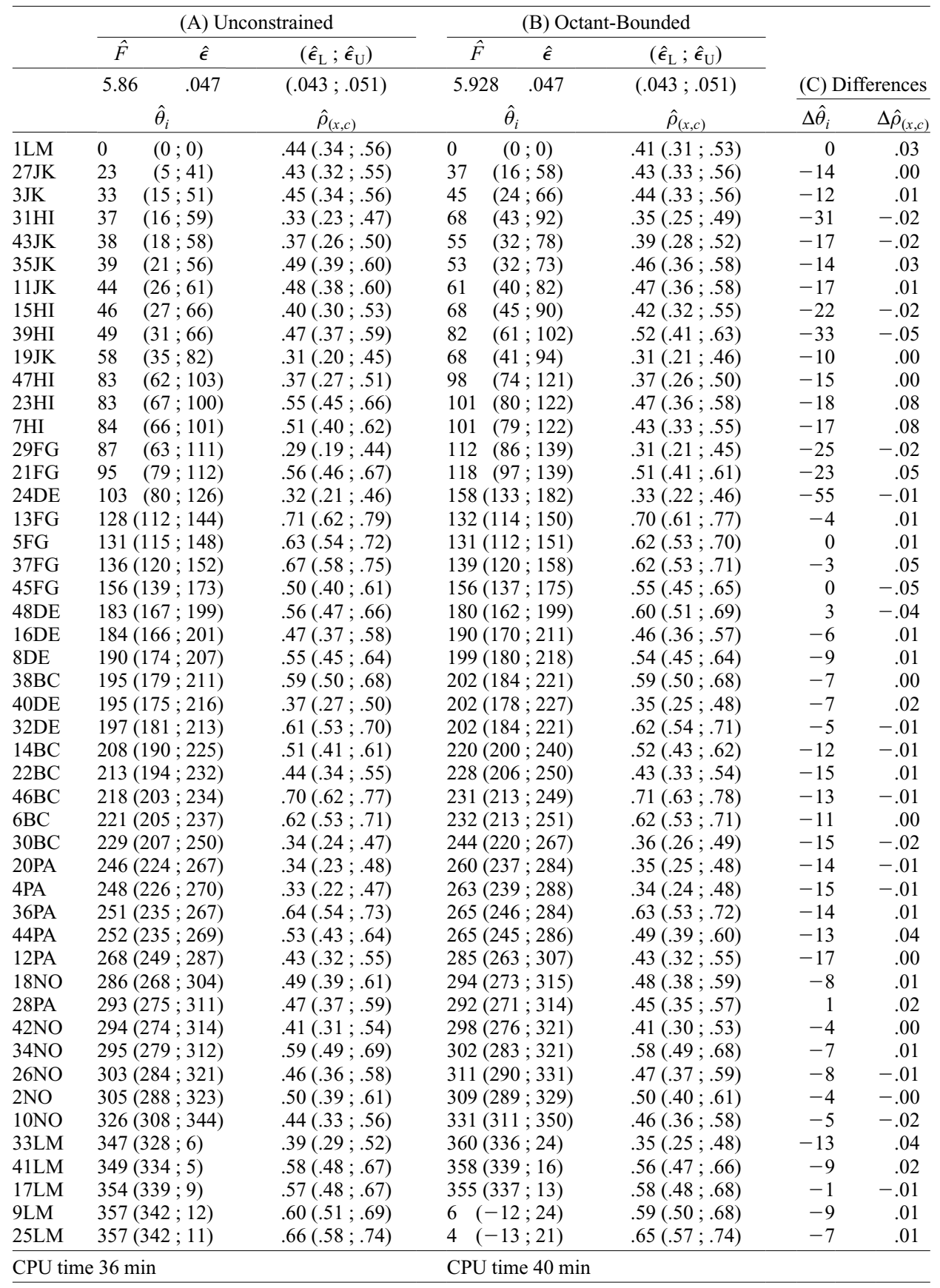




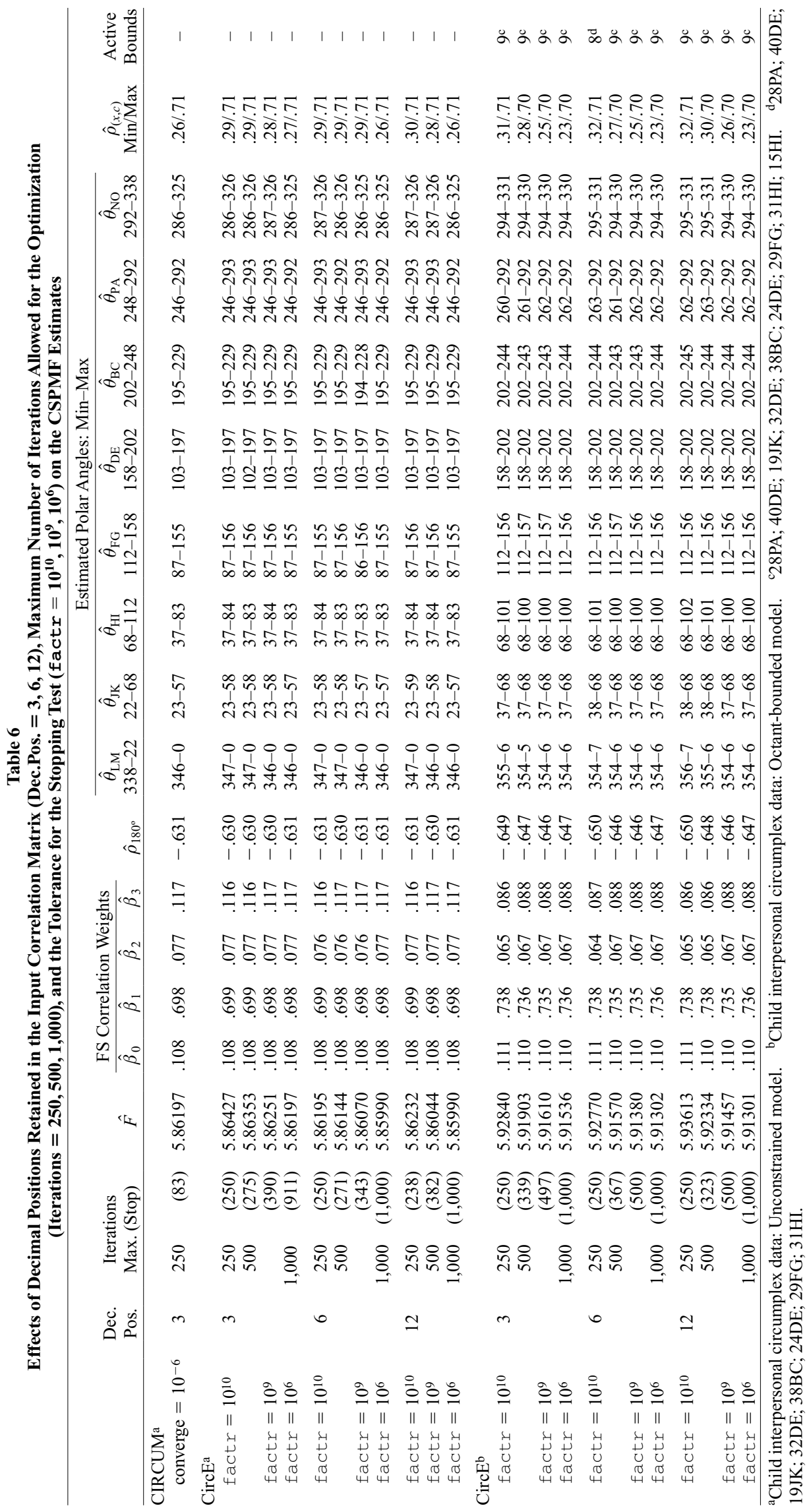


In this model, the parameter vector of $\theta_{i}$ is required to satisfy inequality constraints, since the values of parameters over and under a prespecified bound are undesirable. Importantly, the estimator vector no longer has an asymptotic multivariate normal distribution (Shapiro, 1989), and the goodness-of-fit test statistic no longer has an asymptotic $\chi^{2}$ distribution (Shapiro, 1985) if some estimates at the time that the program converges on a solution are on the boundary of what constitutes theoretically possible values. The appropriate distribution for the test statistic is then a mixture of $\chi^{2}$ distributions with different degrees of freedom. In particular, the true asymptotic distribution lies between $\chi^{2}$ distributions with $d=p(p+1) / 2-q$ and with $d+s$ degrees of freedom, where $s$ is the number of active inequality constraints at the time of convergence. CircE prevents parameter estimates from iterating beyond the range of theoretically possible values and then reports (see Appendix B) the $\chi^{2}$ test and fit indices corresponding to these two extreme cases (the values in square brackets). The confidence interval for the parameters is uncorrected for the nonnormal component introduced by the active bounds. Nevertheless, the amplitude of the confidence intervals can be used for a rough judgment of the estimates' precision, especially when a large number of items and a relatively small number of active bounds are at stake.

\section{Non-Positive-Definite Matrices}

A distressing error message to read from the output of a structural equation modeling program is that a covariance/ correlation matrix is not positive definite (Schumacker \& Lomax, 1996). Technically speaking, if the determinant of a matrix is 0 , the matrix is known as a singular or non-positive-definite matrix. This also means that the inverse of the matrix does not exist. Statistically speaking, a covariance/correlation matrix is positive definite if all of its eigenvalues are greater than 0 . Under these conditions, numerous computations related to covariance/correlation matrices either cannot be generated or cannot be trusted. There are three ${ }^{8}$ situations in which a researcher may receive a message that a matrix is not positive definite: Either (1) the input covariance or correlation matrix being analyzed, (2) the model-implied covariance matrix, $\Sigma$, or (3) the variance/covariance matrix of the parameters is not positive definite. If any of these matrices is found to be not positive definite, both CIRCUM and CircE will issue an error message. The three situations can be very different in terms of their causes and cures. A more in-depth treatment of how to deal with matrices that are not positive definite is contained in the seminal work of Wothke (1993). What follows are ways to recover from such failures using the CircE program.

Sample covariance/correlation matrix is nonpositive-definite. A non-positive-definite input matrix may signal a perfect linear dependency (collinearity) among the observed variables. Collinearity may be a result of one of the following factors: (1) a composite or totalscore variable that is a sum of two or more component variables, all of which are included in the covariance matrix; (2) outliers or extreme observations; (3) a sample size that is smaller than the number of observed variables; or (4) error in transcribing a matrix. When the sample size is small, a sample covariance or correlation matrix may fail to be positive definite merely due to sampling fluctuation. Moreover, non-positive-definiteness of the input matrix typically occurs when the pairwise (rather than listwise) deletion method is used for handling missing data.

Analyses of such input matrices using CIRCUM fail to converge on a final set of parameter estimates. Remington, Fabrigar, and Visser (2000) found that the correlation matrix reported by Block (1957; see the supplemental materials, file Block.rda) was unanalyzable, and thus did not include it in the analyses reported in their article. The error message reported by CIRCUM highlights that the problem of fitting a CSPMF to Block's data occurs at the early stage of starting value computation. CircE offers a way to sidestep this problem without actually trying to discern its cause: In fact, at this stage, the image factor analysis (IFA) cannot be carried out, since the inverse of the non-positivedefinite Block's matrix has negative values on the diagonal (Jöreskog, 1969, p. 54), as shown in Listing 5.

To overcome this problem, CircE offers an alternative way to obtain initial parameters estimation, based on principal factor analysis (PFA):

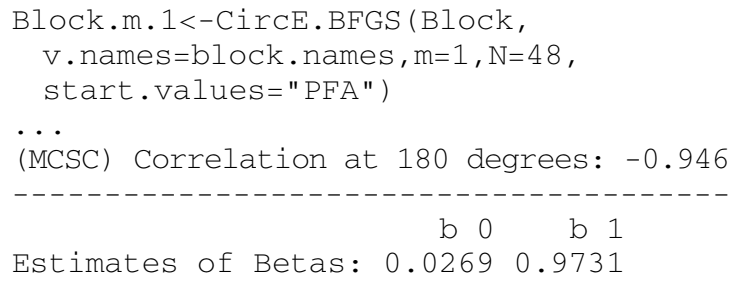

The PFA supplies likely starting values; in fact, replicating the analysis performed in Example 1 by setting start. values $=$ PFA produces the same results. The number of

Listing 5

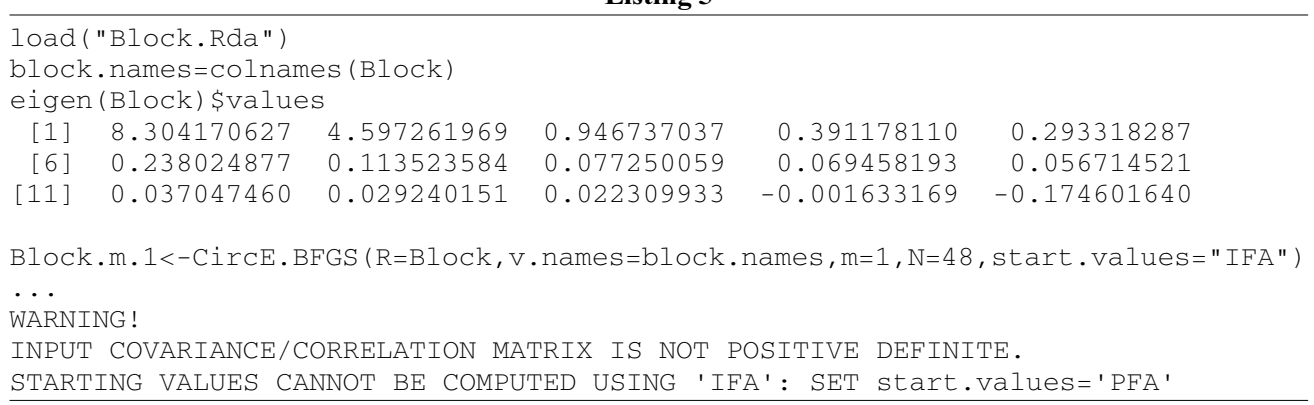


iterations required by the program to converge may be different; moreover, the direction of the ordering of variables from the reference variable around the circumference (but not the order itself) could be completely reversed. Such a reversal would not change any other aspect of the solution, including the fit of the model, the MCSC, the communalities, or the distances among the variables on the circle. Thus, to aid in comparison of the solutions for different data, models, and/or starting values, the estimates of polar angles in the output are present in both possible directions.

Singular model-implied $\Sigma$ matrix. The second situation in which a non-positive-definite covariance/ correlation matrix might be a problem is in the estimation of the covariance matrix constructed from the model specified by the researcher. Generally, this happens when the model is not properly specified. In our practice of working with CircE, the model-implied matrix (Equation 2) may become singular during a failure of the nonlinear optimization algorithm (negative discrepancy function values). Let us consider again Block's matrix; now trying to estimate a CSPMF model with $m=3$ :

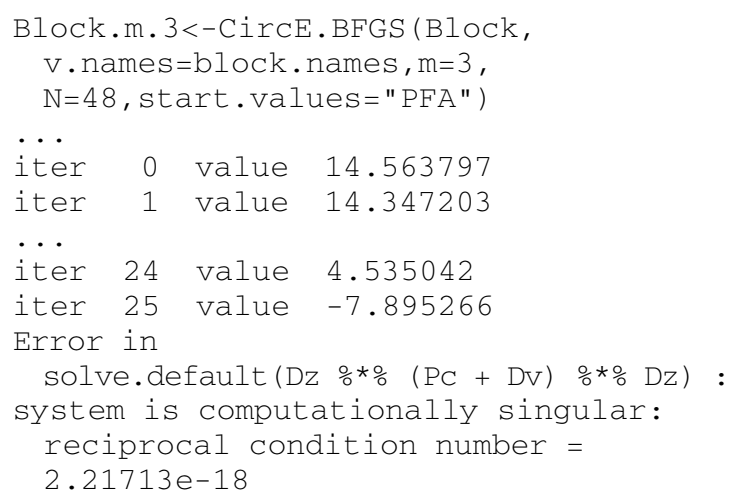

A way to recover from such convergence failures is to adjust the parameter $1 \mathrm{~mm}$ that controls the behavior of the optimization algorithm. This parameter is set by default to the number of free parameters $q$ (e.g., $1 \mathrm{~mm}=47$ for Block's matrix). If the algorithm fails to converge, $1 \mathrm{~mm}$ should be decreased within the recommended range $3 \leq 1 \mathrm{~mm} \leq 20$ (Zhu et al., 1994), as shown in Listing 6.

Nevertheless, in this case we have clearly overspecified the number of $\beta \mathrm{s}$ in the Fourier correlation function (Equation 3), since $\beta_{2}$ and $\beta_{3}$ attain the lower bound of zero. So the failure in the convergence is due mainly to an incorrect specification of the model.

Singular Hessian matrix. The third situation in which a non-positive-definite matrix can occur involves the estimation of the covariance matrix for the parameters. In our confirmatory circumplex analysis with Browne's CSPMF, the main reason for the existence of a singular parameter covariance matrix is related to a problem of identification. A model is underidentified if there are more parameters to be estimated than there are elements in the covariance matrix. The mathematical properties of underidentified models prevent a unique solution to the parameter estimates and prevent goodness-of-fit tests, since the degrees of freedom are negative. Considering the way in which the CSPMF is conceptualized, the likelihood of identification problems is minimal. In fact, by constraining the angular distance, the communality, and/or the MCSC, users can increase the degrees of freedom of the model. However, in working with few observed variables, the selection of an excessive number of free parameters $(m)$ in the correlation function (Equation 3 ) could lead to an underidentified model.

Let us consider the following correlation matrix proposed by Guttman (1954, p. 282; see also Browne, 1992, p. 470, and the supplemental materials, file Verbal.rda), which gives the intercorrelations between six verbal ability tests (Listing 7).

Attempting to fit an unconstrained CSPMF with $m=5$ (an arbitrary value that does not seem too high) free pa-

Listing 6

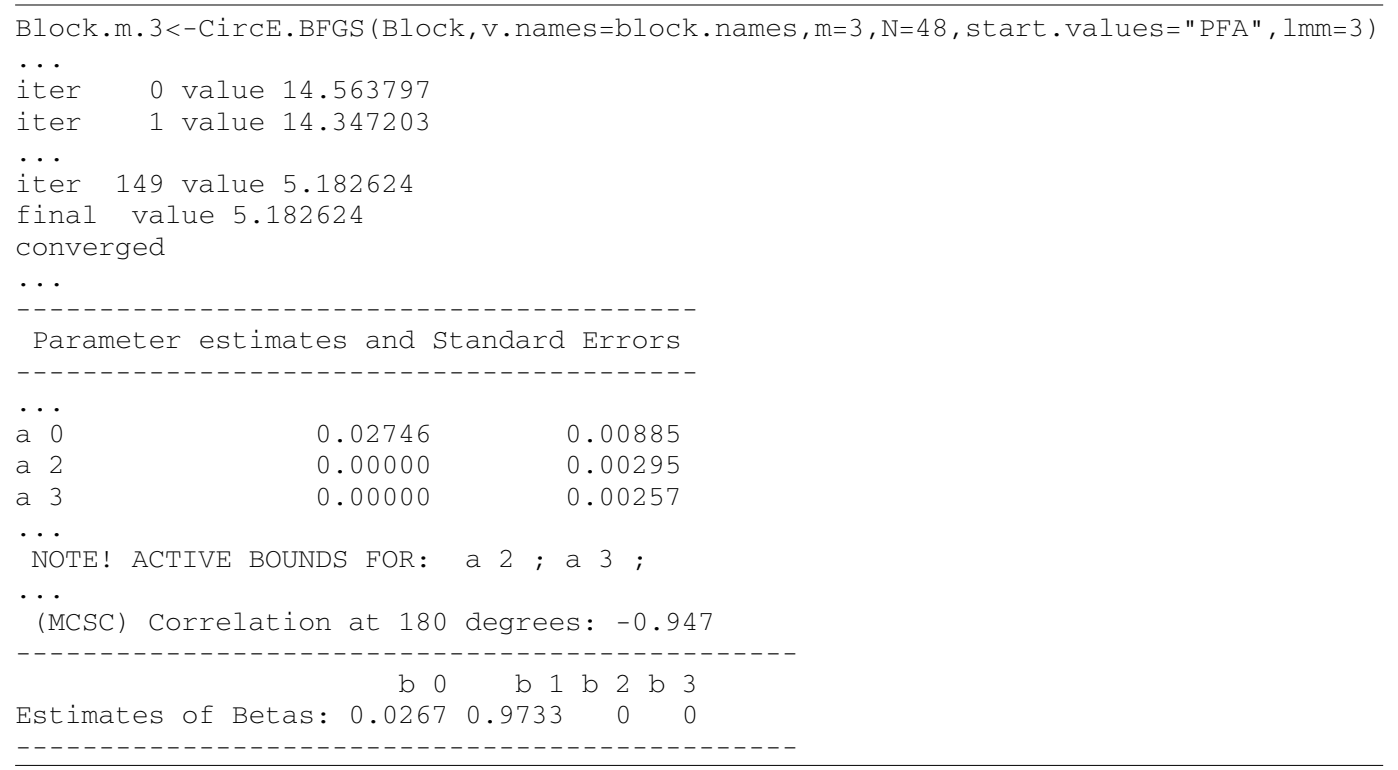


Listing 7

\begin{tabular}{|c|c|c|c|c|c|c|c|}
\hline \multicolumn{8}{|c|}{$\begin{array}{l}\text { load ("Verbal.rda" ) } \\
\text { verbal. names=colnames (Verbal) } \\
\text { Verbal }\end{array}$} \\
\hline & Spelling & Punctuation & Grammar & Vocabulary & Literature & Foreign & Literature \\
\hline Spelling & 1.000 & 0.621 & 0.564 & 0.476 & 0.394 & & 0.389 \\
\hline Punctuation & 0.621 & 1.000 & 0.742 & 0.503 & 0.461 & & 0.411 \\
\hline Grammar & 0.564 & 0.742 & 1.000 & 0.577 & 0.472 & & 0.429 \\
\hline Vocabulary & 0.476 & 0.503 & 0.577 & 1.000 & 0.688 & & 0.548 \\
\hline Literature & 0.394 & 0.461 & 0.472 & 0.688 & 1.000 & & 0.639 \\
\hline Foreign Literature & 0.389 & 0.411 & 0.429 & 0.548 & 0.639 & & 1.000 \\
\hline
\end{tabular}

rameters in the correlation function, the degrees of freedom of the model, $d=6(6+1) / 2-(5+5+6+6)=$ $21-22=-1$, are negative, and the program alerts the user to an identification problem, asking whether or not to continue, as shown in Listing 8.

Where CIRCUM simply quits the computation, we have programmed CircE to continue, which results in a singular Hessian matrix. Of course, this has been done mainly for demonstration purposes; in this situation, the researcher is well advised to type NO and to ensure the identification of the model by reducing $m$ or using equality constraints (equal. $\mathrm{com}=\mathrm{TRUE}$ and/or equal. ang = TRUE).

\section{Discussion and Concluding Remarks}

There may be a considerable interval before methodological progress in the field of data analysis is commonly employed in practice. One reason is certainly the highly technical nature of these discussions. Even if psychometricians and statisticians have paid great attention to the circumplex structure of data, their results are rarely acknowledged by the majority of social and personality psychologists. A more effective driver for the dissemination of new forms of analysis is the availability of dedicated statistical programs. The CSPMF proposed by Browne (1992) has been implemented in a program, CIRCUM, that has been widely used by social and personality psychologists, even though it is not integrated in the most-used statistical software packages. This success is partially due to research (e.g., Fabrigar et al., 1997; Remington et al., 2000) that has sought to bridge the gap between exquisitely statistical literature and field studies. Yet the importance of an analytic tool that can be directly applied, is easy to use, and can be adjusted to the researcher's needs cannot be denied. In terms of ease of use, CIRCUM is certainly an accessible program, even though it is available only as an executable file and is not part of any common data analysis software. Therefore its use is subject to entering data into another statistical program to obtain a covariance/correlation matrix, which then has to be manually inserted into CIRCUM via a file text that includes some parameters necessary to set the model. As regards flexibility of use, all of the main needs for the analysis of circumplex models are fulfilled with this program. Yet it does not cover seemingly less important needs, such as the opportunity to directly use the analysis

Listing 8

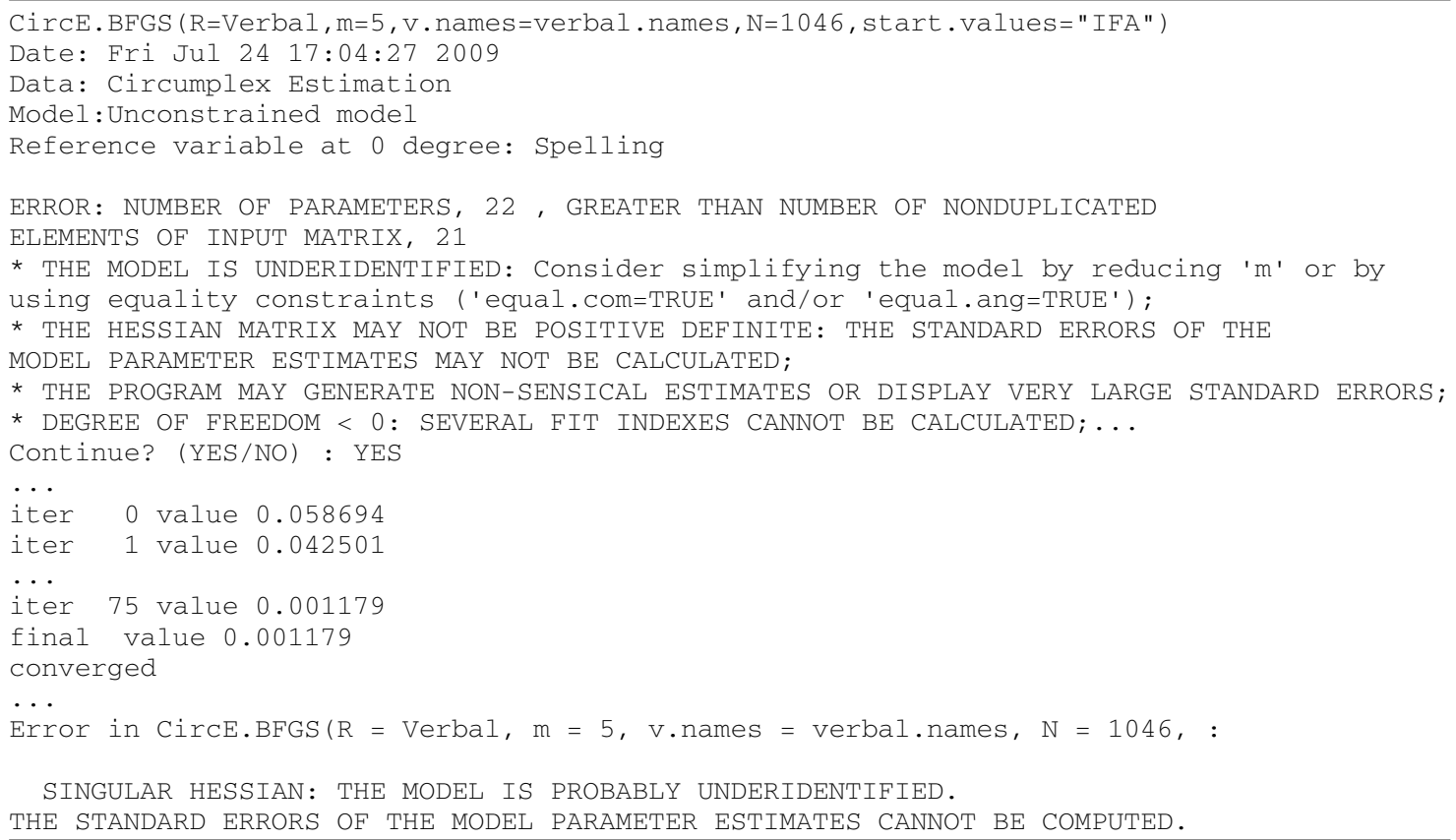


results for further processing. Indeed, graphical representations of variables along the circumference and of the estimated FS correlation function must be produced by external software, into which the necessary information must be entered to obtain the graphs. The quality of the final result is then directly proportional to the user's ability to stray from the default graphical settings of the software used.

Although these are minor problems if CIRCUM is used only occasionally, exploratory analyses, above all in terms of questionnaire items, would be much simplified if (1) the statistical routine were integrated in the software used for data entry, (2) the results of the analysis could be represented in a graph, and (3) the chance to constrain the estimates could be extended to items that are conceptually linked to higher-order variables, in order to assess the empirical tenability of their expected circular organization. In the present article, we have presented an R package for fitting circumplex models, an alternative to the widely used CIRCUM. It is "alternative" in the senses that the results provided by CircE are convergent with those obtained by CIRCUM and that the options for constraining the model allowed by CIRCUM are present also in CircE. Moreover, CircE has the compelling advantages of being integrated in one of the most widely used packages for statistical computing and of providing users with graphical functions, as well as providing users with the ability to specify bounded estimations of polar angles, which is particularly appropriate in questionnaire item analysis. As seen in the child interpersonal circumplex example, each item can be constrained to fall within a specific $45^{\circ}$ arc; that is, items are constrained to be distributed across the eight octants LM to JK, which cover the whole circumference and represent different possible DOM/LOV combinations. This approach allows testing of whether an expected organization of items into scales is empirically robust, whereas other presently available statistical packages do not allow for testing such specific circular distributions of an item. All of these features are programmed in an open language that is easy to check and modify with minor programming effort. A future enhancement would be to expand CircE's computational capabilities to multiple-group models and alternative fitting functions, such as the asymptotically distribution-free discrepancy function (Browne, 1984; Yung \& Bentler, 1994). The rapidity with which this will be accomplished will depend on users' interest.

\section{AUTHOR NOTE}

We thank Michael W. Browne for useful documentation he provided and Michael Siegal for comments on an earlier draft. We also thank the three anonymous reviewers, who helped us improve this article. Correspondence concerning this article should be addressed to M. Grassi, Department of Psychology “G. Kanizsa," Via Sant'Anastasio 12, 34134, Trieste, Italy (e-mail: grassi.mic@gmail.com).

\section{REFERENCES}

Anderson, T. W. (1960). Some stochastic process models for intelligence test scores. In K. J. Arrow, S. Karlin, \& P. Suppes (Eds.), Mathematical methods in the social sciences, 1959 (pp. 205-220). Stanford: Stanford University Press.
BLOCK, J. (1957). Studies in the phenomenology of emotions. Journal of Abnormal \& Social Psychology, 54, 358-363. doi:10.1037/h0040768

Bollen, K. A. (1989). Structural equations with latent variables. New York: Wiley.

Browne, M. W. (1982). Covariance structures. In D. M. Hawkins (Ed.), Topics in applied multivariate analysis (pp. 72-141). Cambridge: Cambridge University Press.

BRowne, M. W. (1984). Asymptotically distribution-free methods for the analysis of covariance structures. British Journal of Mathematical \& Statistical Psychology, 37, 62-83.

BRowne, M. W. (1992). Circumplex models for correlation matrices. Psychometrika, 57, 469-497. doi:10.1007/BF02294416

Browne, M. W., \& Cudeck, R. (1992). Alternative ways of assessing model fit. Sociological Methods \& Research, 21, 230-258.

Browne, M. W., \& Du ToIT, S. H. C. (1992). Automated fitting of nonstandard models. Multivariate Behavioral Research, 27, 269-300. doi:10.1207/s15327906mbr2702_13

Byrd, R. H., Lu, P., Nocedal, J., \& Zhu, C. (1995). A limited memory algorithm for bound constrained optimization. SIAM Journal on Scientific Computing, 16, 1190-1208. doi:10.1137/0916069

Di Blas, L., Grassi, M., Luccio, R., \& Momentè, S. (2008). How children rate children: Structural continuity of the circumplex model of interpersonal behavior in late childhood. Manuscript submitted for publication.

Fabrigar, L. R., Visser, P. S., \& Browne, M. W. (1997). Conceptual and methodological issues in testing the circumplex structure of data in personality and social psychology. Personality \& Social Psychology Review, 1, 184-203. doi:10.1207/s15327957pspr0103_1

Fan, X., \& Sivo, S. A. (2007). Sensitivity of fit indices to model misspecification and model types. Multivariate Behavioral Research, 42, 509-529.

GutTMAn, L. (1954). A new approach to factor analysis: The radex. In P. F. Lazarsfeld (Ed.), Mathematical thinking in the social sciences (pp. 258-348). Glencoe, IL: Free Press.

Hu, L., \& Bentler, P. M. (1999). Cutoff criteria for fit indexes in covariance structure analysis: Conventional criteria versus new alternatives. Structural Equation Modeling, 6, 1-55.

JöRESKOG, K. G. (1969). Efficient estimation in image factor analysis. Psychometrika, 34, 51-75. doi:10.1007/BF02290173

Liu, D. C., \& Nocedal, J. (1989). On the limited memory BFGS method for large scale optimization. Mathematical Programming, 45, 503-528. doi:10.1007/BF01589116

MacCallum, R. C., Browne, M. W., \& Sugawara, H. M. (1996). Power analysis and determination of sample size for covariance structure modeling. Psychological Methods, 1, 130-149. doi:10.1037/1082 $-989 X .1 .2 .130$

R Core Team Members, DebRoy, S., Bivand, R., et al. (2008). foreign: Read data stored by Minitab, S, SAS, SPSS, Stata, Systat, dBase, .. (R package version 0.8-26). Vienna: R Foundation for Statistical Computing.

R Development Core Team (2008). R: A language and environment for statistical computing (ISBN 3-900051-07-0). Vienna: R Foundation for Statistical Computing.

Remington, N. A., Fabrigar, L. R., \& Visser, P. S. (2000). Reexamining the circumplex model of affect. Journal of Personality \& Social Psychology, 79, 286-300. doi:10.1037/0022-3514.79.2.286

Schumacker, R. E., \& Lomax, R. G. (1996). A beginner's guide to structural equation modeling. Mahwah, NJ: Erlbaum.

ShapIRo, A. (1985). Asymptotic distribution of test statistic in the analysis of moment structures under inequality constraints. Biometrika, 72, 133-144. doi:10.1093/biomet/72.1.133

Shapiro, A. (1989). Asymptotic properties of statistical estimators in stochastic programming. Annals of Statistics, 17, 841-858. Available at http://projecteuclid.org/euclid.aos/1176347146.

SPSS INC. (1999). SPSS base 10.0 for Windows user's guide. Chicago: Author.

STEIGER, J. H., \& Lind, J. M. (1980, June). Statistically based tests for the number of common factors. Paper presented at the annual meeting of the Psychometric Society, Iowa City, IA.

WigGINs, J. S. (1979). A psychological taxonomy of trait-descriptive terms: The interpersonal domain. Journal of Personality \& Social Psychology, 37, 395-412. doi:10.1037/0022-3514.37.3.395 


\section{APPENDIXA (Continued)}

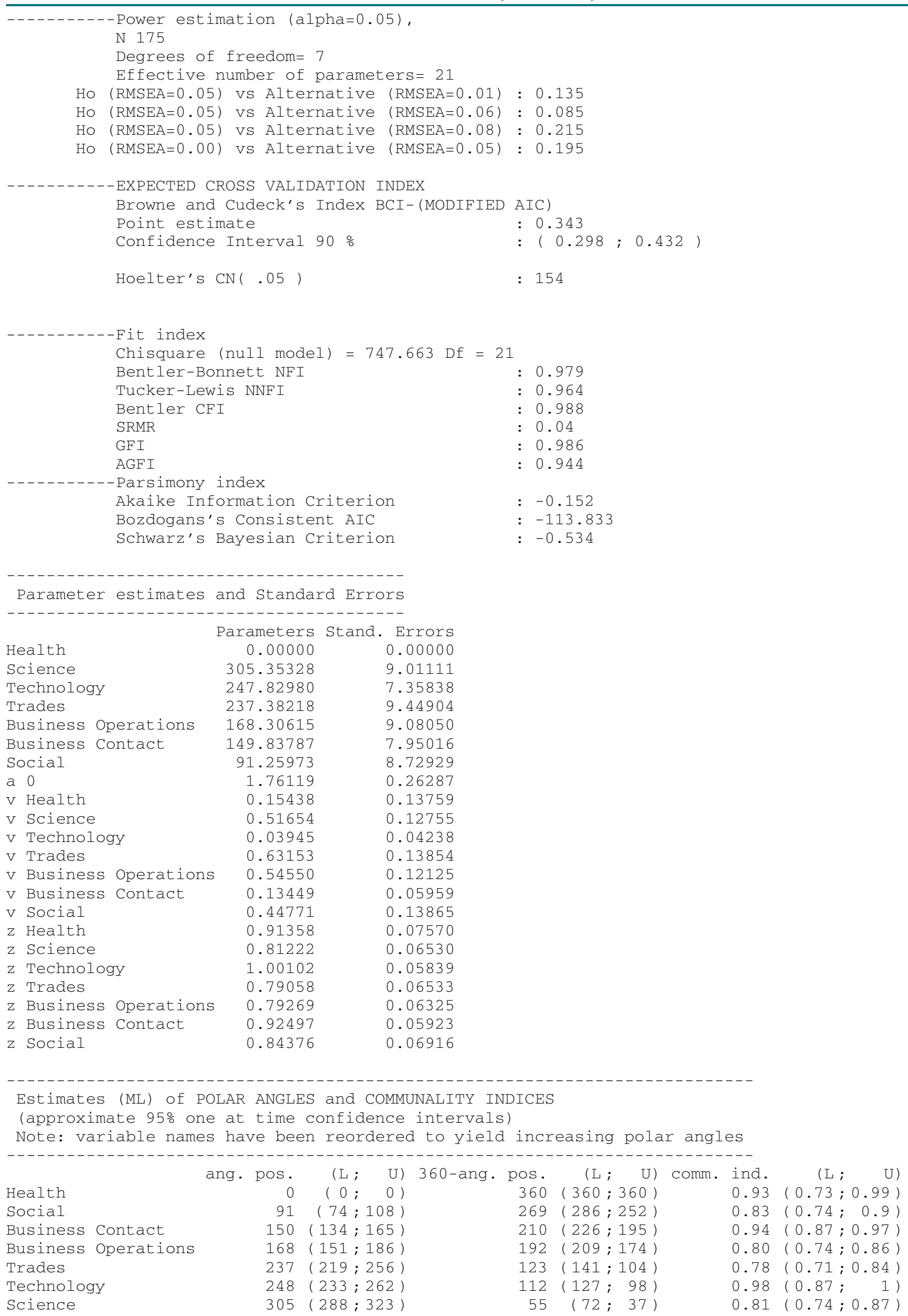




\section{APPENDIXA (Continued)}

(MCSC) Correlation at 180 degrees: 0.276

b $0 \quad$ b 1

Estimates of Betas: 0.63780 .3622

CPU Time for optimization 2.412 sec. ( 0 min.)

\section{Reproduced and Residual Matrices}

residual. CircE(Voc. unconstr, digits=3)

\begin{tabular}{|c|c|c|c|c|c|c|c|c|c|}
\hline & Health & Science & Technology & Trades & Business & Operations & Business & Contact & Social \\
\hline Health & 1.000 & 0.654 & 0.453 & 0.251 & & 0.122 & & 0.218 & 0.496 \\
\hline Science & 0.654 & 1.000 & 0.644 & 0.440 & & 0.158 & & 0.210 & 0.264 \\
\hline Technology & 0.453 & 0.644 & 1.000 & 0.757 & & 0.551 & & 0.570 & 0.366 \\
\hline Trades & 0.251 & 0.440 & 0.757 & 1.000 & & 0.493 & & 0.463 & 0.202 \\
\hline Business Operations & 0.122 & 0.158 & 0.551 & 0.493 & & 1.000 & & 0.754 & 0.471 \\
\hline Business Contact & 0.218 & 0.210 & 0.570 & 0.463 & & 0.754 & & 1.000 & 0.650 \\
\hline Social & 0.496 & 0.264 & 0.366 & 0.202 & & 0.471 & & 0.650 & 1.000 \\
\hline
\end{tabular}

Reproduced Correlation Matrix

\begin{tabular}{|c|c|c|c|c|c|c|c|c|c|}
\hline & Health & Science & Technology & Trades & Business & Operations & Business & Contact & Social \\
\hline Health & 1.000 & 0.640 & 0.458 & 0.323 & & 0.212 & & 0.284 & 0.487 \\
\hline Science & 0.640 & 1.000 & 0.663 & 0.492 & & 0.243 & & 0.235 & 0.228 \\
\hline Technology & 0.458 & 0.663 & 1.000 & 0.763 & & 0.555 & & 0.541 & 0.249 \\
\hline Trades & 0.323 & 0.492 & 0.763 & 1.000 & & 0.483 & & 0.480 & 0.219 \\
\hline Business Operations & 0.212 & 0.243 & 0.555 & 0.483 & & 1.000 & & 0.741 & 0.481 \\
\hline Business Contact & 0.284 & 0.235 & 0.541 & 0.480 & & 0.741 & & 1.000 & 0.645 \\
\hline Social & 0.487 & 0.228 & 0.249 & 0.219 & & 0.481 & & 0.645 & 1.000 \\
\hline
\end{tabular}

Reproduced Common Score Correlation Matrix

\begin{tabular}{|c|c|c|c|c|c|c|c|c|c|}
\hline & Health & Science & Technology & Trades & Business & Operations & Business & Contact & $\begin{array}{r}\text { Social } \\
0.630\end{array}$ \\
\hline Health & 1.000 & 0.847 & 0.501 & 0.443 & & 0.283 & & 0.325 & \\
\hline Science & 0.847 & 1.000 & 0.832 & 0.774 & & 0.373 & & 0.308 & 0.3 \\
\hline Technology & 0.501 & 0.832 & 1.000 & 0.994 & & 0.704 & & 0.587 & 0.3 \\
\hline Trades & 0.443 & 0.774 & 0.994 & 1.000 & & 0.767 & & 0.653 & 0.3 \\
\hline usiness Operations & 0.283 & 0.373 & 0.704 & 0.767 & & 1.000 & & 0.981 & 0.7 \\
\hline usiness Contact & 0.325 & 0.308 & 0.587 & 0.653 & & 0.981 & & 1.000 & 0.8 \\
\hline ocial & 0.630 & 0.338 & 0.306 & 0.337 & & 0.719 & & 0.827 & \\
\hline
\end{tabular}

$\begin{array}{cccrr}\text { Ratios of Reproduced Variances to Input Variances } & & \\ \text { Health } & \text { Science } & \text { Technology } & \text { Trades } & \text { Business Operations } \\ 0.963 & 1.000 & 1.042 & 1.020 & 0.971\end{array}$ Social 1.031

Residual Matrix (CORRELATION)

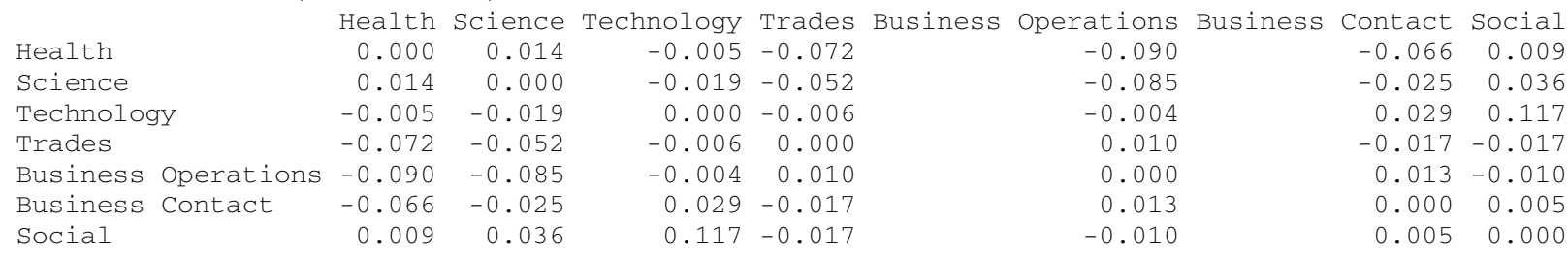

Residuals

Min. 1st Qu. Median Mean 3rd Qu. Max. $\begin{array}{llllll}-0.090 & -0.019 & -0.004 & -0.010 & 0.009 & 0.117\end{array}$ 


\section{APPENDIXA (Continued)}

\begin{tabular}{|c|c|c|c|c|c|}
\hline \multicolumn{6}{|c|}{ Standardized Residuals } \\
\hline $\begin{array}{r}\text { Min } \\
-0.090\end{array}$ & $\begin{array}{l}1 \text { st } Q u \\
-0.019\end{array}$ & $\begin{array}{l}\text { Median } \\
-0.004\end{array}$ & $\begin{array}{r}\text { Mean } \\
-0.010\end{array}$ & $\begin{array}{r}3 \text { rd Qu. } \\
0.009\end{array}$ & $\begin{array}{r}\text { Max. } \\
0.117\end{array}$ \\
\hline
\end{tabular}

\section{APPENDIX B}

\section{Reduced Output of the Octant-Bounded Model}

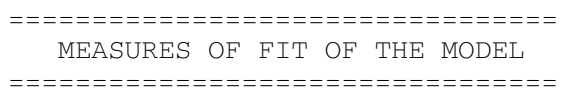

NOTE: 9 PARAMETERS ( 28PA ; 40DE ; 19JK ; 32DE ; 38BC ; 24DE ; 29FG ; 31HI ; 15HI ; ) ARE ON A BOUNDARY.

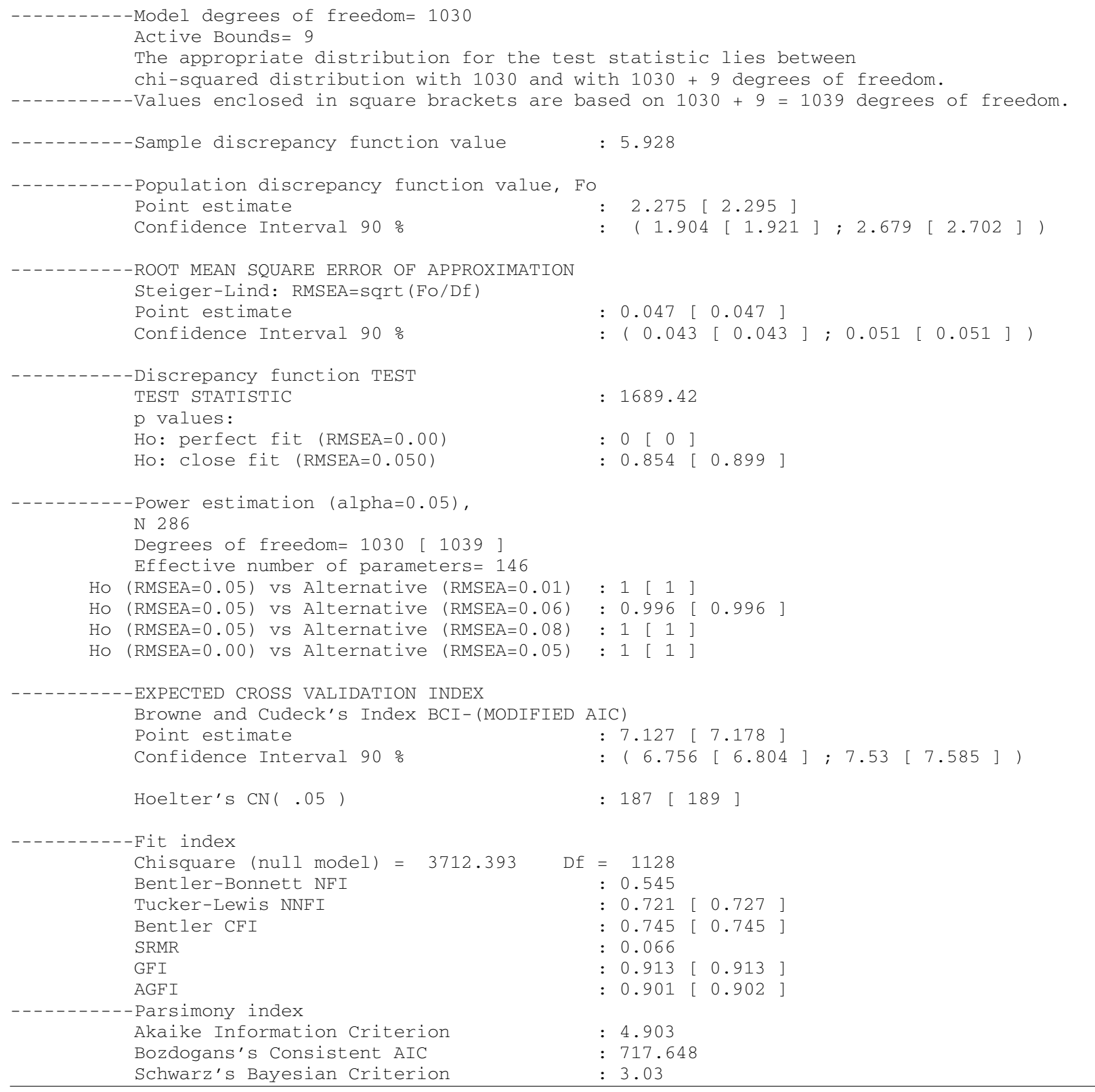

